\title{
16th Meeting of the Interuniversity Institute of Myology (IIM) - Assisi (Italy), October 17-20, 2019: Foreword, Program and Abstracts
}

\author{
Davide Gabellini (1), Antonio Musarò (2) \\ (1) Gene Expression and Muscular Dystrophy Group, Division of Genetics and Cell Biology, \\ IRCCS San Raffaele Scientific Institute, Milano, Italy; (2) DAHFMO-Unit of Histology and \\ Medical Embryology, Laboratory Affiliated to "Istituto Pasteur Italia - Fondazione Cenci \\ Bolognetti”, Sapienza University of Rome, Rome, Italy \\ This article is distributed under the terms of the Creative Commons Attribution Noncommercial License (CC BY-NC 4.0) which permits \\ any noncommercial use, distribution, and reproduction in any medium, provided the original author(s) and source are credited.
}

\begin{abstract}
The 16th Meeting of the Interuniversity Institute of Myology (IIM), October 17-20, 2019, Assisi, Italy brought together scientists, pharma and patient organization representatives discussing new results on muscle research. Internationally renowned Keynote speakers presented advances on muscle development, homeostasis, metabolism, and disease. Speakers selected among submitted abstracts presented their new, unpublished data in seven scientific sessions. The remaining abstracts were showcased in two poster sessions. Young trainees where directly involved in the selection of keynote speakers, the organizing scientific sessions and roundtables discussions tailored to the interests of their peers. A broad Italian, European and North-American audience participated to the different initiatives. The meeting allowed muscle biology researchers to discuss ideas and scientific collaborations aimed at better understanding the mechanisms underlying muscle diseases in order to develop better therapeutic strategies. The active participation of young trainees was facilitated by the friendly and inclusive atmosphere, which fostered lively discussions identifying emerging areas of myology research and stimulated scientific cross-fertilization. The meeting was a success and the IIM community will continue to bring forward significant contributions to the understanding of muscle development and function, the pathogenesis of muscular diseases and the development of novel therapeutic approaches. Here, we report abstracts of the meeting illustrating novel results of basic, translational, and clinical research, which confirms that the Myology field is strong and healthy.
\end{abstract}

Key Words: muscle development, homeostasis, regeneration, metabolism, wasting, stem cells, epigenetics, proof of concept, translational, ex-vivo, in-vivo, clinical trials.

Eur J Transl Myol 2020; 30 (3), 9345. doi: 10.4081/ejtm.2020.9345

The Interuniversity Institute of Myology (IMM) was founded in 2003 with the mission to promote efforts to further the understanding of normal and pathological aspects of muscle formation, activity and repair. Training of young fellows and promotion of international collaborations between researchers, clinicians and patient associations are key tools for development of therapeutic treatments for neuromuscular diseases.

The 16th IIM Meeting took place in Assisi, from October 17 to 20, 2019. The meeting brought together investigators performing muscle research in Universities and Research Centers, as well as representatives of pharma and patient organizations, form Italy and abroad. The meeting covered, in a multidisciplinary way, the different aspects of muscle biology, including development, homeostasis, regeneration, pathogenesis, pathology and clinics of neuromuscular disorders, fostering discussions to promote new ideas, research lines, and collaborations. There were four Keynote Lectures by Olivier Pourquié (Harvard Stem Cell Institute Boston-USA), Bente Klarlund Pedersen (University of Copenhagen-Denmark), Alessandra Sacco (Sanford Burnham La Jolla-USA) and Bénédicte Chazaud (Institut NeuroMyoGène Lyon-France).

Skeletal muscle is one of the most plastic organs of our body able to adopt specific phenotypic properties in response to a wide range of factors, including physical activity, injury, stimulation by motor neurons, oxygen and nutrient supply, and changes in hormone levels. In this context, fiber type is an essential determinant of muscle function and alteration in fiber composition represents a major component in the muscle degeneration 
associated with muscle diseases. ${ }^{1}$ Leading experts of the muscle field, along with speakers selected from submitted abstracts, provided important insights into the extracellular agonists, receptors, protein kinases, intermediate molecules, transcription factors, and epigenetic mechanisms involved in the modulation of specific cellular responses in embryonic and adult myocytes/myofibers..$^{2-5}$

Olivier Pourquié demonstrated that, mimicking key signaling events leading to muscle formation in the embryo, it is possible to efficiently produce striated, millimeter-long muscle fibers together with satellite-like cells from human pluripotent stem cells (ES/iPS), avoiding the requirement for genetic modifications or cell sorting. Bente Klarlund Pedersen discussed the important role of skeletal muscle as an endocrine organ, which has the capacity to produce hundreds of myokines, providing a conceptual basis for understanding how muscles communicate with other organs such as adipose tissue, liver, pancreas, bones, and brain. Alessandra Sacco discussed the dynamics of muscle stem cells during tissue growth and regeneration, whereas Bénédicte Chazaud discussed the roles of macrophages in normal skeletal muscle regeneration versus muscle disease.

Muscle regeneration recapitulates many aspects of embryonic myogenesis and it is an important homeostatic process of adult skeletal muscle, which, after development, retains the capacity to regenerate in response to appropriate stimuli, activating the muscle compartment of stem cells, namely satellite cells, and other precursor cells. ${ }^{3,6-8}$ Skeletal muscle stem cells (MuSC), also known as satellite cells, are the main source of skeletal muscle growth and regeneration. Within their native tissue microenvironment, or stem cell niche, they are exposed to signals emanating from neighboring cells, as well as the extracellular space that instruct their cell fate decisions, whether to undergo self-renewal or commitment and differentiation upon cell division. By utilizing genetic and pharmacological tools, Sacco and co-workers have identified STAT3 signaling as a critical regulator of MuSC fate decisions.

The inflammatory response of injured skeletal muscle plays an important and critical role in muscle homeostasis and regeneration and consists in the recruitment of specific myeloid cell populations within the injured area. ${ }^{3,7,8}$ Macrophages rapidly increases within 24 hours after injury, are the predominant inflammatory cell type within the injured area, detectable at the level of perimysium and epimysium. Because of their high versatility and their impact on their environment, they sustain both the mounting and the dampening of the inflammatory response. In a context of adult tissue damage, that is post-injury skeletal muscle regeneration, accumulating lines of evidence of the beneficial and pleiotropic roles of macrophages in tissue repair indicate that inflammation should not be considered as a bad or detrimental process. Conversely, as discussed by Chazaud, it should be viewed as a dynamic process of which sequential steps must be tightly coordinated in space and time to be fully efficient to support skeletal muscle regeneration.

Based on the submitted abstracts, thirty five contributions were selected for Oral Communications that were grouped in seven Sessions chaired by young trainees: 1 . Genetic and epigenetic alterations in muscle dystrophies and myopathies; 2. Satellite cells and muscle regeneration in healthy muscle and in diseases; 3 . Biophysics and E-C coupling in the pathophysiology of neuromuscular diseases; 4. Signaling in muscle growth, homeostasis and disease; 5. Metabolic alterations and muscle diseases; 6. Muscle fibrosis, sarcopenia and cachexia; 7. Therapeutic approaches for muscle diseases. Twenty seven poster presentations, always on display during the entire Meeting, completed the program. Presentations provided breakthroughs in the muscle field, at a fundamental and therapeutic level, and stimulated lively and exciting scientific discussions, which were continued through spontaneous gatherings of several groups at coffee breaks and after lunch and dinner leading to the development of new collaborations.

On the first evening of the meeting, there were three Roundtable Discussions organized by young trainees and moderated by the invited speakers and selected members of the IIM. The participants discussed about new ideas concerning muscle development \& regeneration; inflammation, metabolism; oxidative muscle damage. The roundtables allowed to discuss muscle research in an informal way, over food and drinks provided by local producers. On the second evening of the meeting, guided tours were organized to the historic section of the city of Montefalco and the "Cantina Lungarotti" winery, terminating with a wine tasting and banquet dinner. Finally, an Award Ceremony was held on the last evening of the meeting to congratulate Marco Rosina, Martina Sandonà and Valentina Taglietti, winners of prices for best Poster, Oral and Active Participation awards, respectively, selected by an international panel composed by the Invited Speakers and key members of the IIM.

For the first time, the IIM Meeting was joined with a High Training Course on "Advanced Myology", organized together with the University of Perugia and reserved to young trainees. The highlight of the Course was on October 20th with lectures on muscle development and response to exercise by international speakers taking place in Palazzo Bernabei, a beautiful 1400 building located in front of the St. Francis Basilica. The meeting and the course were very successful and instrumental to foster new ideas and facilitate collaborations among the different stakeholders.

Taken together, the attendees of the IIM and of the PaduaMuscleDays (PMDs, a meeting more oriented to advanced Translational Myology) and the authors of papers e-printed in the European Journal of Translational Myology (EJTM) are a substantial part of the international community of Myologists. ${ }^{9,10,11}$ Here, the 
abstracts of the 16th IIM Meeting show that this community will continue to deliver major contributions to the understanding and treatment of skeletal muscle diseases.

\author{
List of acronyms \\ EJTM - European Journal of Translational Myology \\ ES/iPS - satellite-like cells from human pluripotent stem \\ cells \\ IMM - Interuniversity Institute of Myology \\ MuSC - Skeletal muscle stem cells \\ PMDs - PaduaMuscleDays
}

\section{Author's contributions}

Authors equally contributed to write the manuscript.

\section{Acknowledgements}

Funding This typescript was sponsored by PAGEpress, Pavia, Italy and the $\mathrm{A} \& \mathrm{C} \mathrm{M}-\mathrm{C}$ Foundation for Translational Myology, Padova, Italy.

\section{Conflict of Interest}

The authors declare they have no financial, personal, or other conflicts of interest.

\section{Ethical Publication Statement}

We confirm that we have read the Journal's position on issues involved in ethical publication and affirm that this report is consistent with those guidelines.

\section{Corresponding Authors}

Davide Gabellini, Gene Expression and Muscular Dystrophy Group, Division of Genetics and Cell Biology, IRCCS San Raffaele Scientific Institute, Milano, Italy.

ORCID Id: 0000-0002-3811-4121

Email: gabellini.davide@hsr.it

Antonio Musarò, DAHFMO-Unit of Histology and Medical Embryology, Laboratory Affiliated to Istituto Pasteur Italia - Fondazione Cenci Bolognetti, Sapienza University; Center for Life Nano Science@Sapienza, IIT, Rome, Italy.

ORCID Id: 0000-0002-2944-9739

E-mail: antonio.musaro@uniroma1.it

\section{References}

1. Scicchitano BM, Dobrowolny G, Sica G, Musarò A. Molecular Insights into Muscle Homeostasis, Atrophy and Wasting. Curr Genomics 2018 Aug;19(5):356-369. doi: 10.2174/1389202919666 18101153911

2. Gabellini D, Green MR, Tupler R. When enough is enough: genetic diseases associated with transcriptional derepression. Curr Opin Genet Dev 2004 Jun;14(3):301-7. doi: 10.1016/j.gde.2004. 04.010

3. Musarò A. Muscle Homeostasis and Regeneration: From Molecular Mechanisms to Therapeutic Opportunities. Cells 2020 Sep 4;9(9):E2033. doi: 10.3390/cells9092033..

4. Neguembor MV, Jothi M, Gabellini D. Long noncoding RNAs, emerging players in muscle differentiation and disease. Skelet Muscle 2014;4(1):8. doi: 10.1186/2044-5040-4-8.

5. Neguembor MV, Gabellini D. In junk we trust: repetitive DNA, epigenetics and facioscapulohumeral muscular dystrophy. Epigenomics 2010;2(2):271-87. doi: 10.2217/epi.10.8.

6. Forcina L, Miano C, Pelosi L, Musarò A. An Overview about the Biology of Skeletal Muscle Satellite Cells. Curr Genomics 2019;20(1):24-37. doi: 10.2174/1389202920666190116094736.

7. Forcina L, Cosentino M, Musarò A. Mechanisms Regulating Muscle Regeneration: Insights into the Interrelated and Time-Dependent Phases of Tissue Healing.Cells 2020;9(5):1297. doi: 10.3390/cells 9051297.

8. Scicchitano BM, Sica G, Musarò A. Stem Cells and Tissue Niche: Two Faces of the Same Coin of Muscle Regeneration. Eur J Transl Myol 2016 Nov 25;26(4):6125. doi: 10.4081/ejtm.2016.6125. eCollection 2016 Sep 15.

9. Barberi L, Scicchitano BM, Musaro A. Molecular and Cellular Mechanisms of Muscle Aging and Sarcopenia and Effects of Electrical Stimulation in Seniors. Eur J Transl Myol 2015;25(4):231-6. doi: 10.4081/ejtm.2015.5227

10. Carraro U. 2020PMD, 30-years of Translational Mobility Medicine at the time of COVID-19 outbreak: Last-minute forewords from the editor. Eur J Transl Myol 2020;30(1):8966. doi: 10.4081/ejtm.2019.8966

11. Carraro U. Thirty years of translational research in Mobility Medicine: Collection of abstracts of the 2020 Padua Muscle Days. Eur J Transl Myol 2020;30(1):8826. doi: 10.4081/ejtm.2019.8826

Submission: September 11, 2020 Accepted for pubblication: September 11, 2020 


\section{iim

\section{6th IIM Meeting}

1 St HIGH TRAINING COURSE IN ADVANCED MYOLOGY Assisi 17-20 October 2019

\section{TOPICS}

\section{Pathogenesis and Therapies of Neuromuscular Diseases}

- Biophysics and E-C coupling

- Genetics and epigenetic

- Muscle stem cells and regenerative medicine

- Muscle wasting and cachexia

- Exercise

- Signaling and metabolism

- Therapeutic approaches

\section{Keynote Lectures}

Bénédicte Chazaud (Institut NeuroMyoGène Lyon-France)

Bente Klarlund Pedersen (University of Copenhagen-Denmark)

Olivier Pourquié (Harvard Stem Cell Institute Boston-USA)

Alessandra Sacco (Sanford Burnham La Jolla-USA)

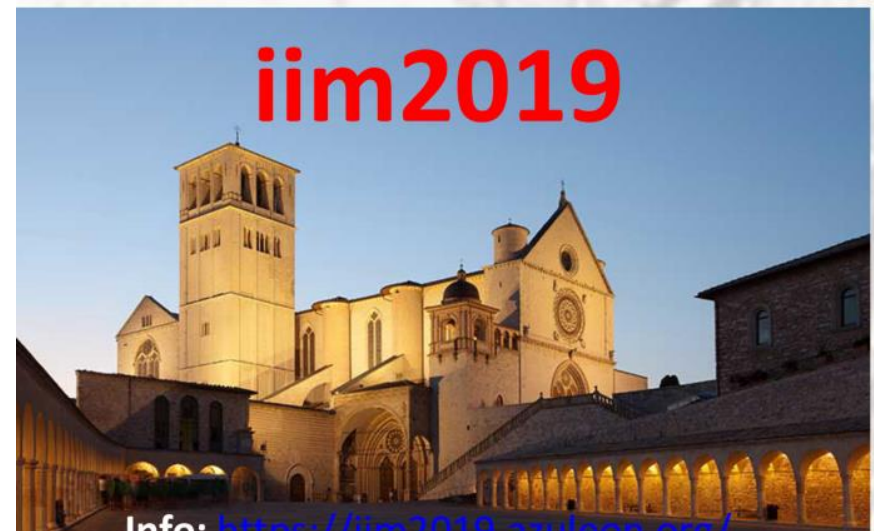

Info:

secretary: fisiologia@unich.it

\section{Venue}

Hotel II Cenacolo (Assisi-Italy)

http://www.hotelcenacolo.com/

\section{Scientific Committee}

Barbieri E, Blaauw B, Fulle S, Gabellini D, Gargioli C, Grassi F, Musarò A, Mammucari C, Moresi V, Puri PL, Sampaolesi M, Sandri M, Sorci G. 


\section{With the support of}
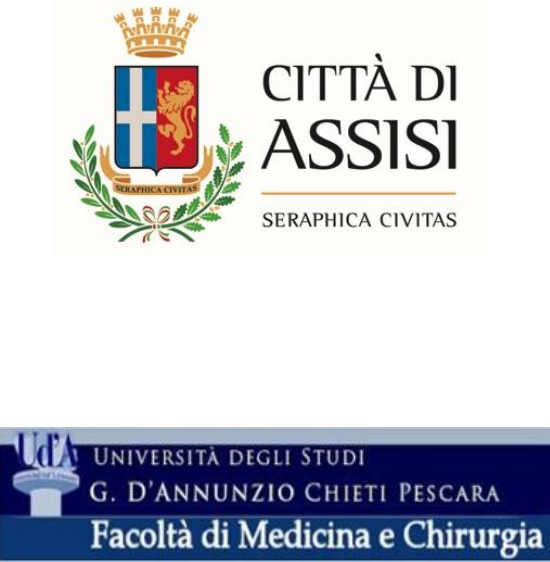

Facoltà di Medicina e Chirurgia
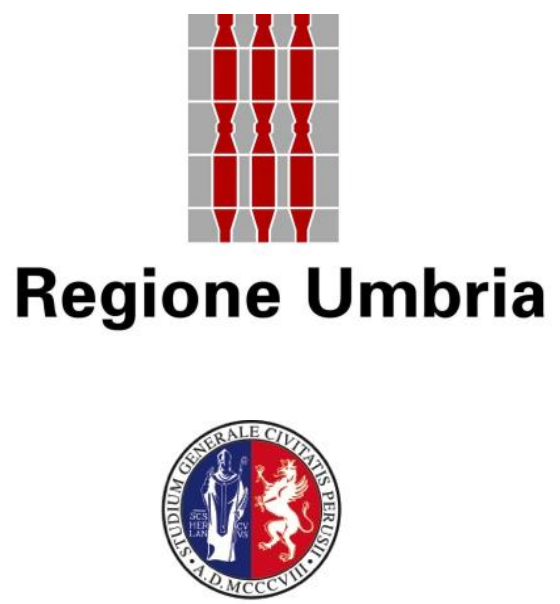

UNIVERSITÀ DEGLI STUDI

DI PERUGIA

\section{Q $\mathrm{BCC}$ Spello e Bettona}
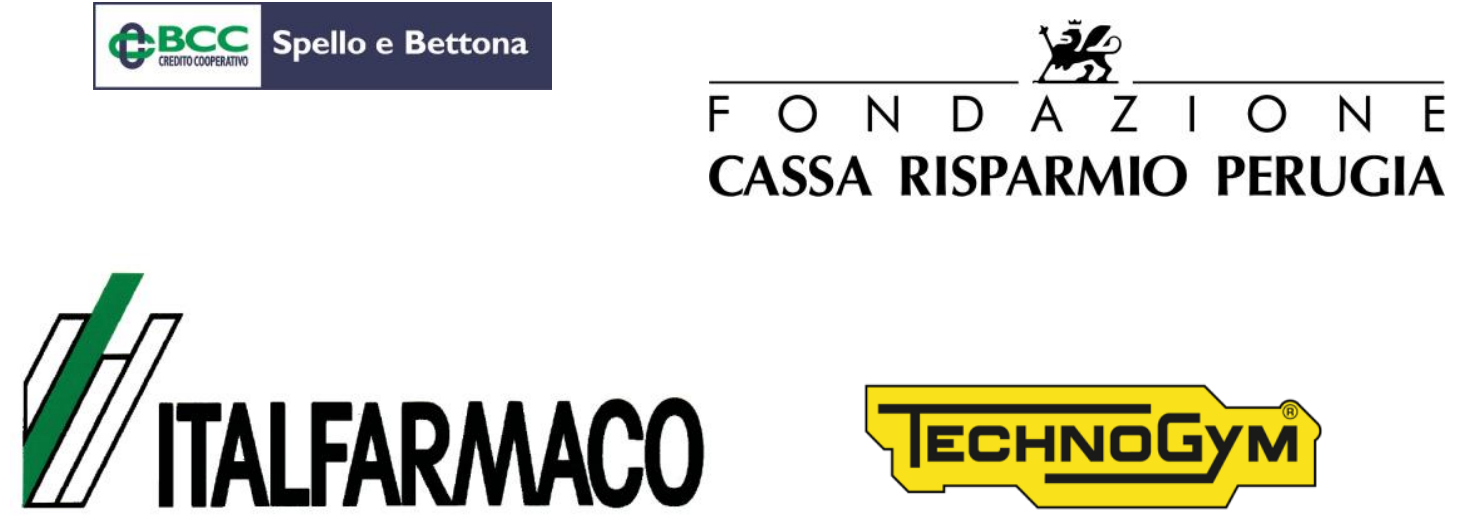

\section{Sponsored by}

fPRODOTTIGIANNI

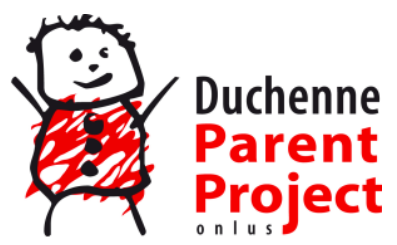

\section{AFMTELETHON \\ CURE THROUCH INNOVATION}


16th IIM Meeting (2019) - Foreword \& Abstracts

Eur J Transl Myol 2020; 30 (3), 9345. doi: 10.4081/ejtm.2020.9345

\section{INVITED SPEAKERS}


16th IIM Meeting (2019) - Foreword \& Abstracts

Eur J Transl Myol 2020; 30 (3), 9345. doi: 10.4081/ejtm.2020.9345

\section{LECTURE 1 Alessandra Sacco (Sanford Burnham La Jolla-USA)}

\section{Dynamics of muscle stem cells during tissue growth and regeneration}

Skeletal muscle stem cells (MuSC), also known as satellite cells, are the main source of skeletal muscle growth and regeneration. Within their native tissue microenvironment, or stem cell niche, they are exposed to signals emanating from neighboring cells as well as the extracellular space that instruct their cell fate decisions, whether to undergo self-renewal or commitment and differentiation upon cell division. By utilizing genetic and pharmacological tools, we have identified STAT3 signaling as a critical regulator of MuSC fate decisions. STAT3 is a transcription factor that is rapidly activated upon tissue injury, and promotes MuSC commitment. By performing RNA sequencing analysis, we provided evidence that STAT3 signaling promotes this transition by regulating mitochondrial respiration through the secreted factor Fam3a. We are currently developing tools for transient inhibition of this pathway to bias MuSC towards expansion and promote tissue maintenance and repair. We are further exploring the role of extracellular matrix proteins (ECM) in regulating MuSC behavior. ECM proteins play a major role in sequestering or presenting growth factors to MuSC, as well as regulating tissue architecture and stiffness. We have shown that the ECM protein Tenascin-C, abundant in developing muscles and upon tissue damage, stimulates MuSC expansion during postnatal muscle growth and adult tissue repair, and we are currently utilizing proteomic approaches to define the Tenascin- $C$ interactome and the downstream networks mediating its effects on MuSC. Finally, we are utilizing multicolor lineage tracing approaches in vivo to track the dynamics of clonal heterogeneity in the MuSC compartment, and we have recently shown that upon repeated tissue injury the clonal heterogeneity of MuSC is progressively reduced, unveiling significant changes in MuSC composition in response to stress. The integration of molecular profiling and cell fate mapping in vivo will improve our understanding of how skeletal muscle tissues are maintained and identify novel therapeutic tools and targets for promoting MuSC function and potentiate tissue repair in chronic conditions such as muscle-wasting diseases and aging.

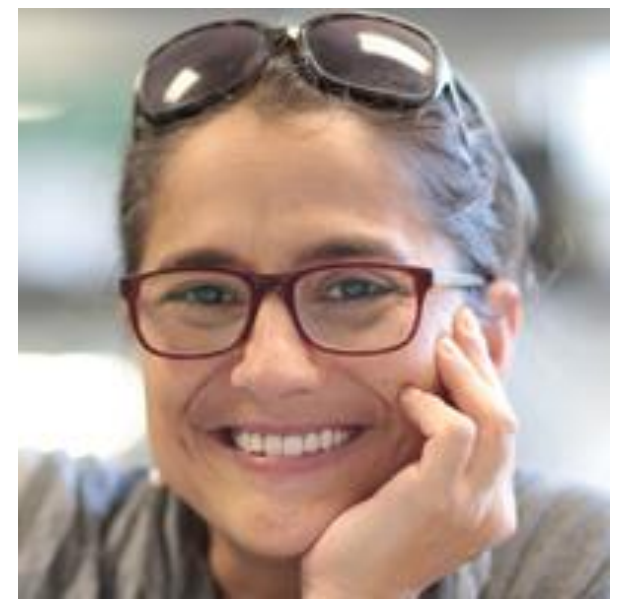

Alessandra Sacco is Associate Professor of Development, Aging and Regeneration Program at Sanford Burnham Prebys Medical Discovery Institute, La Jolla, CA, USA. Since 2012 she also actively participates to the Sanford Burnham Prebys Graduate Program of Biological Sciences, and since 2014 she serves as Associate Dean of Curriculum for the Program. The research of her team focuses on skeletal muscle stem cells (MuSC) and their role in homeostatic maintenance and tissue repair. The Sacco laboratory identified STAT3 signaling as a major regulator of MuSC function. They have shown that STAT3 signaling promotes MuSC activation and commitment through regulation of mitochondrial function. Given the chronic activation of this signaling pathway in several muscle diseases, the team is developing tools for its transient inhibition, to promote tissue repair. Recently, her group pioneered multicolor lineage tracing studies in muscles in vivo to track the dynamics of clonal heterogeneity in the MuSC compartment. They demonstrated that upon repeated tissue injury the clonal heterogeneity of MuSC is progressively reduced, unveiling significant changes in MuSC composition in response to stress. The goal of this research is to define the networks regulating MuSC fate decisions, and through manipulation of the microenvironment or the sensitivity of MuSC to external signals, to develop approaches to be utilized for regenerative medicine for muscle diseases. 
16th IIM Meeting (2019) - Foreword \& Abstracts

Eur J Transl Myol 2020; 30 (3), 9345. doi: 10.4081/ejtm.2020.9345

\section{LECTURE 2 Bente Klarlund Pedersen (University of Copenhagen-Denmark)}

\section{Muscle as an endocrine organ}

Skeletal muscle works as an endocrine organ with the capacity to produce hundreds of myokines. This finding provides a conceptual basis for understanding how muscles communicate with other organs such as adipose tissue, liver, pancreas, bones, and brain. Some myokines are thought to induce anti-inflammatory responses with each bout of exercise and other mediate long-term exercise-induced improvements in cardiovascular risk factors, having an indirect anti-inflammatory effect. Myokines also mediate anticancer effects and contribute to regulate metabolism and cognitive function. The myokine IL-6 is released into the blood during exercise and it has been shown that IL- 6 has multiple immunologic and metabolic effects. Recent advances regarding the physiology of IL- 6 will be discussed. Human studies show that IL-6 infusion delays gastric emptying, reduces postprandial glucose concentrations and reduces insulin secretion, whereas experimental studies suggest a role for IL-6 in appetite regulation. Evidence is also accumulating for a central role of IL-6 in training-induced loss of visceral adipose tissue mass in humans. Moreover, recent experimental studies in mice show that voluntary exercise suppresses tumor growth through epinephrine- and IL-6-dependent mobilization and redistribution of cytotoxic NK cells. It has been known for a while that IL-6 is a pleiotropic molecule, however, recent advances suggest that the physiological roles of IL-6 involve multiple aspects of metabolism as well as a role in tumor defense.

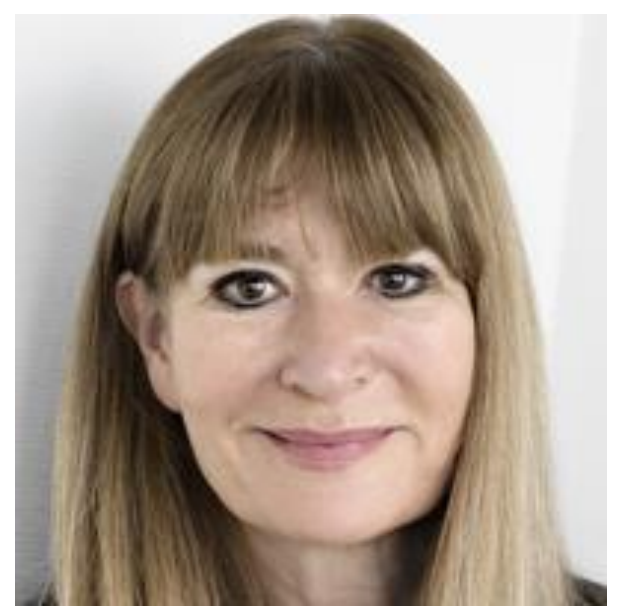

Bente Klarlund Pedersen, MD MDSc, is Professor of Integrative Medicine and a specialist in infectious diseases and internal medicine. She is the Director of the Centre of Inflammation and Metabolism (CIM) and the Centre for Physical Activity Research (CFAS) funded by TrygFonden. CIM and CFAS count 17 senior researchers/postdocs, $17 \mathrm{PhD}$ students, 21 other academic and technical personnel, 5 pre-graduate students and an administration of 5 persons (http://aktivsundhed.dk). She has supervised $47 \mathrm{PhD}$ projects and been a mentor of 5 doctoral theses. The research group has identified skeletal muscle as an endocrine organ that produces and releases so-called "myokines". The identification of myokines provides a conceptual basis for understanding how muscles communicate with other organs. Through translational research, the aim is to develop targeted exercise training regimes for specific disease groups by applying a translational strategy: "from bedside to bench and back". BKP has had many positions of trust and is a member of the Royal Danish Academy of Sciences and Letters. BKP has more than 650 scientific publications, > 41.000 citation and her "H"-index is 107 (Web of Science). 


\section{LECTURE 3 Bénédicte Chazaud (Institut NeuroMyoGène Lyon-France)}

Roles of macrophages in normal skeletal muscle regeneration versus muscle disease

Inflammation after a tissue damage encompasses several sequential phases including: (1) the mounting of the inflammatory response, characterized by the infiltration of immune cells to the site of injury and the release of proinflammatory effectors; (2) the resolution of inflammation, characterized by a shift from a proinflammatory environment to the establishment of the anti-inflammatory phase of inflammation; and (3) tissue repair/regeneration including angiogenesis, matrix remodeling and return to homeostasis. Almost all immune cell types participate in this inflammatory process. However, macrophages are present in high number and during all the sequences of the inflammatory response. Because of their high versatility and their impact on their environment, they sustain both the mounting and the dampening of the inflammatory response. In a context of adult tissue damage, that is post-injury skeletal muscle regeneration, accumulating lines of evidence of the beneficial and pleiotropic roles of macrophages in tissue repair indicate that inflammation should not be considered as a bad or detrimental process. Conversely, it should be viewed as a dynamic process of which sequential steps must be tightly coordinated in space and time to be fully efficient to support skeletal muscle regeneration. Particularly, macrophages exert specific actions on muscle stem cells during the sequential phases of myogenesis, as they first support the expansion of muscle stem cells, then their differentiation and fusion into new functional myofibers. Conversely, in chronic degenerative muscle diseases, such as in muscle dystrophies, phenotypes and functions of macrophages are altered, participating to the pathogeny of the disease. Our recent investigations indicate that the inflammatory status of macrophages may be a therapeutic target to alleviate muscle dystrophies.

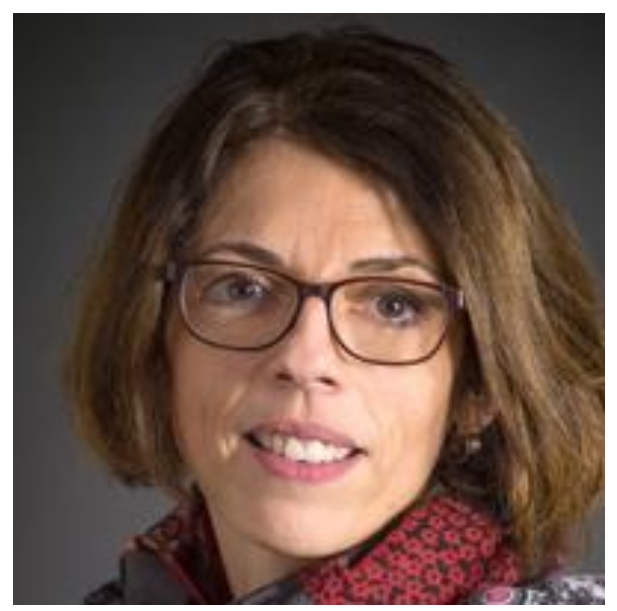

Dr. Bénédicte Chazaud is Director of Research class 1 (eq. of Professor) and Leader of the team "Stem cell environment and skeletal muscle homeostasis" at Institut NeuroMyoGène (INMG) INSERM U1217-CNRS 5310, Université Claude Bernard in Lyon, France. The research of her team is dedicated on the role of environmental cells on adult muscle stem cell behavior in skeletal muscle regeneration and during myopathies. The Chazaud lab was pioneer in bringing the concept of a stromal support for these cells to promote an efficient muscle regeneration. Particularly, they have been extensively studying macrophage biology in this process. They described that beyond their role in innate immunity through scavenging debris, macrophages directly act on muscle stem cells to regulate adult myogenesis. Similarly, while vessels are usually considered only as oxygen and nutriment providers, the work from Dr. Chazaud identified new interactions between endothelial or peri-endothelial cells with muscle stem cells that regulate their fate. The goal of these studies is to decipher the cell interactions that allow a proper myogenesis after muscle injury and to identify the molecular mechanisms underlying these interactions. These investigations are pursued in both normal muscle regeneration as well as in the context of degenerating myopathies. 


\section{In vitro modeling of human muscle development and disease}

Progress toward finding a cure for muscle diseases has been slow because of the absence of relevant cellular models and the lack of a reliable source of muscle progenitors for biomedical investigation. We have developed an optimized serum-free differentiation protocol to efficiently produce striated, millimeter-long muscle fibers together with satellite-like cells from human pluripotent stem cells (ES/iPS) in vitro. By mimicking key signaling events leading to muscle formation in the embryo, this directed differentiation protocol recapitulates the developmental sequence of myogenesis and avoids the requirement for genetic modifications or cell sorting. We engineered a series of human iPS reporter lines including PAX7-YFP, MYOG-YFP and PAX7YFP/MYOD1-Cherry that we used to characterize the differentiation of the myogenic lineage in human myogenic cultures. We are particularly interested in the ontogeny of the human PAX7-expressing lineage that leads to the adult satellite cells. We show that human PAX7-YFP cells produced in vitro exhibit characteristics of fetal satellite cells. We performed single cell RNA sequencing of the PAX7-YFP cells generated in vitro and identified the major steps of the differentiation of this lineage. This work provides a framework to study early stages of human myogenesis which are poorly accessible in the embryo.

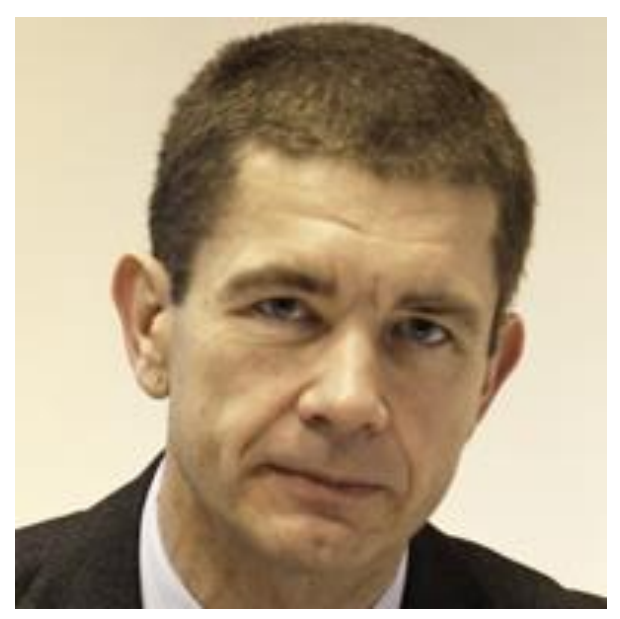

Olivier Pourquie is Professor in the Department of Genetics at Harvard Medical School and Professor of Pathology at the Brigham and Women's Hospital. He is an Associate Member of the Broad Institute and a Principal Faculty member at the Harvard Stem Cell Institute. He was the director of the Institute for Genetics and Molecular and Cellular Biology (IGBMC) in France and before that a Howard Hughes Medical Institute Investigator at the Stowers Institute for Medical Institute in Kansas City. The Pourquie laboratory is a world leader in vertebrate musculo-skeletal axis development. Using chicken and mouse embryos as model systems, they combine developmental biology and genomic approaches to study patterning and differentiation of the precursors of muscles and vertebrae. They also develop quantitative approaches at the interface with physics to study morphogenesis of the vertebral column. While most of this work is being carried out in vivo, they also develop protocols to recapitulate these early developmental processes in vitro using mouse and human embryonic or reprogrammed stem cells. They are also turning to translational approaches, using their understanding of the early development to produce cells of the muscle and vertebral lineages in vitro from pluripotent cells to study human diseases of the musculo-skeletal axis and for cell therapy approaches. Dr. Pourquie graduated as an engineer in France and trained with Nicole Le Douarin in Paris. He authored more than 100 peer-reviewed publications. He is an elected member of the European Molecular Biology Organization and of the Academia Europea. His work on the segmentation clock that controls the periodicity of vertebrae was recognized as one of the milestones in developmental biology of the 20th century by Nature Magazine. 
16th IIM Meeting (2019) - Foreword \& Abstracts

Eur J Transl Myol 2020; 30 (3), 9345. doi: 10.4081/ejtm.2020.9345

\section{PROGRAM SCHEDULE}




\section{Thursday, October $17^{\text {th }}$}

11:30-14:30 Registration

14:45 Welcome and opening of the meeting

15:00-15:40 Lecture 1

Alessandra Sacco (Sanford Burnham La Jolla-USA)

Dynamics of muscle stem cells during tissue growth and regeneration. Chair: Pier Lorenzo Puri

15:40-16:25 Session 1: Genetic and epigenetic alterations in muscle dystrophies and myopathies. Chair: Marianna Cosentino and Samuele Metti

Circulating microRNAs in ALS disease

Irene Casola

Epigenetic regulation of a splicing factor involved in Duchenne Muscular Dystrophy Michela Lisi

New emerging role for HDAC4 on sarcolemma stability in DMD

Alessandra Renzini

\section{6:25-16:55 Coffee break}

16:55-17:30 Welcome from Authorities

17:30-18:45 Session 2: Satellite cells and muscle regeneration in healthy muscle and in diseases. Chair: Ester Di Filippo and Alessandra Renzini

Muscle stem cells from developing children with cerebral palsy: a pilot study Marlies Corvelyn

Physiopathological characterization of the role of MCUb in skeletal muscle regeneration Simona Feno

Splenic Ly6C ${ }^{\text {hi }}$ monocytes are critical players in dystrophic muscle injury and repair Biliana Lozanoska-Ochser

Approaches to delay the progression of Muscular Dystrophy Graziella Messina

Dissecting the secretome during muscle regeneration: role and kinetics of cytokines and extra-cellular vesicles.

Simone Vumbaca

19:30-21:30 Aperitivo Umbro and ROUNDTABLE DISCUSSIONS* 


\section{Friday, October $18^{\text {th }}$}

\section{9:00-9:40 Lecture 2}

Bente Klarlund Pedersen (University of Copenhagen-Denmark)

Muscle as an endocrine organ. Chair: Elena Barbieri

9:40-10:25 Session 3: Biophysics and E-C coupling in the pathophysiology of neuromuscular diseases. Chair: Giosuè Annibalini and Biliana Lozanoska-Ochser

Organ-on-a-chip modelling of the human neuromuscular junction in both physiological and pathological conditions

Ersilia Fornetti

Polyglutamine-expanded androgen receptor alters excitation-contraction coupling machinery and calcium dynamics

Caterina Marchioretti

Transverse Tubule Remodeling Enhances Orai1-dependent $\mathrm{Ca}^{2+}$ Entry in Skeletal Muscle Antonio Michelucci

\section{0:25-10:55 Coffee break}

10:55-12:25 Session 4: Signaling in muscle growth, homeostasis and diseases. Chair: Giorgia Catarinella and Carles Sanchez Riera

The regulation of protein synthesis and muscle plasticity

Ana Georgia Dumitras

Drp1 promotes mitochondrial transport and repositioning in muscle by enhancing kinesin-1 activation

Matteo Giovarelli

The NF-Y regulome in mouse fetal myoblasts reveals a role of SREBP in differentiation Roberto Mantovani

Unveiling novel and specific functions of protein kinase CK2 subunits in skeletal muscle differentiation and fusogenic activity

Giorgia Pallafacchina

Adipogenesis of skeletal muscle Fibro/Adipogenic Progenitors is controlled by the Wnt5a/GSK3/ $\beta$-catenin axis

Alessio Reggio

Evo-Devo approach to study Pax3/7 functions

Valentina Taglietti

13:00 lunch

\section{4:30-16:00 POSTER DISCUSSION}

16:00-16:30 Coffee break

16:40 Bus departure: Guided Tour of Montefalco and Dinner at Cantina Lungarotti 


\section{Saturday, October $19^{\text {th }}$}

\section{9:00-09:40 Lecture 3}

Bénédicte Chazaud (Institut NeuroMyoGène Lyon-France)

Roles of macrophages in normal skeletal muscle regeneration versus muscle disease. Chair: Davide Gabellini

09:40-10:40 Session 5: Metabolic alterations and muscle diseases. Chair: Valentina Taglietti and Marlies Corvelyn

Mitochondrial adaptation in parvalbumin knockout muscle fibers

Gaia Butera

Regulation of oxidative metabolism and substrate preferences in aged skeletal muscle by mitochondrial calcium uptake

Gaia Gherardi

Metabolic reprogramming of Fibro/Adipogenic Progenitors facilitates muscle regeneration Marco Rosina

Identification of a novel TFEB and exercise dependent gene Davide Steffan

\section{0:20-11:10 Coffee break}

11:10-12:55 Session 6: Muscle fibrosis, sarcopenia and cachexia. Chair: Sara Chiappalupi and Giacomo Valli

Direct effects of vitamin $\mathrm{D}_{3}$ (cholecalciferol) on skeletal muscle Maraiza Alves Teixeira

rGDF5, an unexpected treatment against age-related muscle mass loss

Sestina Falcone

The role of vitamin D binding protein in the onset of cancer cachexia - beyond vitamin D transport

Nicoletta Filigheddu

A metabolic shift drives cancer cachexia in myotubes

Michele Mannelli

PIN1: a putative molecular target to protect skeletal muscle against age-related muscle loss Susanna Molinari

Involvement of CerK in skeletal muscle atrophy

Federica Pierucci

Receptor for advanced glycation end-products (RAGE) as a biomarker of muscle wasting in cancer conditions

Aleksandra Vukasinovic

\section{3:00 lunch}


16:00-16:40 IIM young committee invited lecture

Olivier Pourquié (Harvard Stem Cell Institute Boston-USA)

In vitro modeling of human muscle development and disease. Chair: Gaia Gherardi

16:40-17:10 Coffee break

17:10-18:55 Session 7: Therapeutic approaches for muscle diseases. Chair: Giulia Terribile and Emanuele Mocciaro

A novel 3D culture system as an in vitro model for muscle disease Marianna Cosentino

Use of Sertoli cells to treat DMD patients is supported by their immunomodulatory rather than immunosuppressive effect

Sara Chiappalupi

The voice of patients affected by Duchenne and Becker Muscular Dystrophies

Gloria Antonini

Pterostilbene induces autophagic flux in Collagen VI-deficient muscle

Samuele Metti

Inhibition of Acid Sphingomyelinase as Novel Alternative Therapy for Duchenne Muscular Dystrophy

Paulina Melania Roux-Biejat

A novel wet-spinning system for 3D bioprinting of an artificial skeletal muscle tissue Stefano Testa

Hitting DMD With a One-Two Punch: a Novel MAOB/VAP1 Inhibitor Greatly Improves the Pathological Phenotype in mdx Mice

Libero Vitiello

19:00-20:00 IIM General meeting

20:30 Social Dinner - Awards and prizes

22:00 Dance Party

Sunday, October $20^{\text {th }}$

09:30-12:30 High Training lectures*

\section{Departure}

${ }^{*}$ Roundtable discussions and High Training lectures are reserved to participants registered to the High Training Formula. 
16th IIM Meeting (2019) - Foreword \& Abstracts

Eur J Transl Myol 2020; 30 (3), 9345. doi: 10.4081/ejtm.2020.9345

HIGH TRAINING COURSE IN "ADVANCED MYOLOGY”

Thursday October 17, 2019

Hotel II Cenacolo

19:30-21:30 Aperitivo Umbro and ROUNDTABLE DISCUSSIONS

with keynote speakers and selected IIM PIs

Sunday October 20, 2019

Palazzo Bernabei

9:00 bus to Palazzo Bernabei

9:30-9:55

Olivier Pourquie (Harvard Stem Cell Institute Boston-USA)

Skeletal muscle development in vertebrates

9:55-10:00 Discussion

10:00-10:25

Alessandra Sacco (Sanford Burnham La Jolla-USA)

Exercise-induced injury muscle-stem cell responses

10:25-10:30 Discussion

10:30-10:55

Benedicte Chazaud (Institut NeuroMyoGène Lyon-France)

Exercise-induced injury non-muscle cell responses

10:55-11:00 Discussion

11:00-11:25

Bente Klarlund Pedersen (University of Copenhagen-Denmark)

Hormonal responses to exercise

11:25-11:30 Discussion

11:30-11:55

Marco Narici (University of Padova-Italy)

Neuromuscular adaptation to exercise and inactivity

11:55-12:00 Discussion

12:00-12:30

Lunch

13:00 bus to hotel II Cenacolo 


\title{
POSTERS
}

\author{
ALWAYS ON DISPLAY - DISCUSSION: \\ ODD numbers: Friday, October $18^{\text {th }}(14: 30-16: 00)$ \\ EVEN numbers: Saturday, October $19^{\text {th }}(14: 30-16: 00)$
}

P. 01. Lurbinectedin delays the onset of splenomegaly and extends survival of C26 tumorbearing mice

Giorgio Aquila

P. 02. Oxytocin as a physiological anti-cachectic agent?

Alexandra Benoni

P. 03. Role of STAT3-mediated autophagy in driving muscle regeneration during aging

Giorgia Catarinella

P. 04. Novel approaches to counteract aging by physical intervention

Ester Sara Di Filippo

P. 05. Inhibition of N-glycosylation impairs myoblast differentiation and IGF-1 receptor signalling pathways activation

Laura Di Patria

P. 06. Differential expression and epigenetic modulation of TGF- $\beta$ target genes in healthy and DMD skeletal muscle cells

Monica Forino

P. 07. miRNAs as potential molecular biomarkers of cancer cachexia

Lorena Garcia-Castillo

P. 08. SCA1 expression variability governs fibro/adipogenic progenitor (FAP) behaviour

Giulio Giuliani

P. 09. SRF is a mechano-transductor in response to exercise and may play a role in the exercise-mediated rescue of muscle homeostasis in cancer cachexia

Hassani Medhi

P. 10. Sphingolipids regulate VDR expression in skeletal muscle cells Maria Chiara lachini

P. 11. High levels of circulating Interleukin-6 affect the redox balance in skeletal muscle, inducing the expression of inflammatory-related factors

Carmen Miano*, Laura Forcina*

P. 12. The chromatin remodeling protein WDR5 is required for the aberrant expression of DUX4 in facioscapulohumeral muscular dystrophy

Emanuele Mocciaro

P. 13. Extremely low frequency electromagnetic fields modulate the strength in skeletal muscle in sedentary adult mice

Caterina Morabito

P. 14. Inflammation in dystrophic heart and diaphragm: a comprehensive study 
Jacopo Morroni

P. 15. Intracellular attenuation of thyroid hormone influences energy metabolism by reducing mitochondria biogenesis and inducing mitochondrial dysfunction

Annarita Nappi

P. 16. Musclin, a myokine induced by aerobic exercise, retards muscle atrophy during cancer cachexia

Andrea David Re Cecconi

P. 17. The transcription factor NF-Y is required for satellite stem cell proliferation and skeletal muscle tissue repair

Giovanna Rigillo

P. 18. The Thyroid Hormone activating enzyme, Type 2 deiodinase, induces myogenic differentiation by regulating mitochondrial metabolism and reducing oxidative stress.

Serena Sagliocchi

P. 19. Unraveling endurance differences on dystrophy affected muscle groups

Carles Sanchez-Riera

P. 20. Epigenetic tuning of miR in FAP-derived Extracellular vesicles promotes regeneration and inhibits fibrosis in dystrophic muscles

Martina Sandonà

P. 21. Dissecting the role of heterochromatin conformation in muscle aging and sarcopenia Philina Santarelli

P. 22. Evidence supporting a morpho-functional interaction between telocytes and satellite cells in damaged skeletal muscle

Chiara Sassoli

P. 23. Dissecting the possible role of p97 in muscle wasting during cancer

Giulia Terribile

P. 24. The RNA binding protein FRG1 controls transcription landscape regulating muscle maturation and metabolism

Rossella Tupler

P. 25. Effects of aerobic, resistance and combined exercises on 24-hr glucose variability, metabolism and muscle signalling pathways regulation in Type 1 Diabetics

Giacomo Valli

P. 26. N6-Methyladenosine as a novel pharmacological target involved in muscle regeneration

Francesco Millozzi

P. 27. Identification of a phytotherapy formulation to counteract muscle atrophy

Laura Salvadori 


\section{SELECTED TALK ABSTRACTS}

Session 1: Genetic and epigenetic alterations in muscle dystrophies and myopathies Session 2: Satellite cells and muscle regeneration in healthy muscle and in diseases

Session 3: Biophysics and E-C coupling in the pathophysiology of neuromuscular diseases Session 4: Signalling in muscle growth, homeostasis and diseases

Session 5: Metabolic alterations and muscle diseases

Session 6: Muscle fibrosis, sarcopenia and cachexia

Session 7: Therapeutic approaches for muscle diseases 


\subsection{Circulating microRNAs in ALS disease \\ Irene Casola ${ }^{a^{*}}$, Gabriella Dobrowolny ${ }^{*}$, Julie Martone ${ }^{b}$, Elisa Lepore ${ }^{a}$, Irene Bozzonib,c \# , Antonio Musaròa \# a. Institute Pasteur Cenci - Bolognetti, DAHFMO - Unit of Histology and Medical Embryology, La Sapienza University - b. Dept. of Biology and Biotechnology Charles Darwin, La Sapienza University of Rome - c. Centre for Life Nano Science, Istituto Italiano di Tecnologia,} Rome, Italy - * Co - First Authors, \# Co - Corresponding Authors

Amyotrophic Lateral Sclerosis (ALS) is a fatal neurodegenerative disease, characterised by muscle atrophy, weakness, and progressive degeneration of motor neurons, leading to death by respiratory failure within $2 / 5$ years from the onset of clinical symptoms. Diagnosis of ALS relies only on clinical, electrophysiological and neuropathological examination, and is based on the exclusion of alternative related pathologies. Nowadays specific molecular markers for ALS diagnosis or prognosis are not available. MicroRNAs are a class of highly stable molecules, detectable in serum samples, recently proposed as biomarkers for several human diseases, such as cancer and muscular disorders. The aim of the present study was to identify circulating microRNAs as serum molecular markers of ALS disease. Indeed, we performed an NGS analysis on a sub-cohort of patients, and we identified several differentially expressed microRNAs. Moreover, we analysed a subset of microRNAs preferentially expressed in skeletal muscle (myo-miRNAs), involved in myogenesis, and skeletal muscle differentiation and regeneration. Here we provide a longitudinal study on serum samples of 27 spinalonset ALS patients, analysed every three months by absolute RT quantification. We associated serum levels of the identified microRNAs with the disease stage, evaluated by ALS - Health State Scale (ALS-HSS), and we observed that some microRNAs significantly changed during disease progression, while the others were good biomarkers for early and late stages of the disease. Furthermore, since ALS disease progression is variable in terms of velocity and aggressiveness, we divided patients cohort into two subgroups with a different disease progression rate (slow and fast), evaluated by ALS - Functional Rating Scale (ALS-FRS). We identified a group of microRNAs differentially modulated in slow and fast progressing patients, that resulted as good prognostic biomarkers for ALS patients at the moment of the diagnosis. Overall the results of our study can contribute to define a molecular signature of ALS phenotypes, and can allow to better characterise patients to enrol in clinical trials.

\subsection{Epigenetic regulation of a splicing factor involved in Duchenne Muscular Dystrophy}

Michela Lisi ${ }^{1}$, Francesco Castagnetti ${ }^{1}$, Adriano Setti ${ }^{1}$, Alessandro Rosa ${ }^{1,2}$, Valerio Di Carlo ${ }^{3}$, Luciano Di Croce $^{3}$, Olga Sthandier ${ }^{1}$, Julie Martone ${ }^{1}$, Irene Bozzoni ${ }^{1,2,4}$

1. Dept. of Biology and Biotechnology Charles Darwin, Sapienza University - 2. Center for Life Nano Science@Sapienza, Istituto Italiano di Tecnologia - 3. Dept. of Chemistry and Technologies of Drugs, - 4. Institute Pasteur Fondazione Cenci-Bolognetti, Sapienza University of Rome, Italy. - 5. Centre for Genomic Regulation, The Barcelona Institute of Science and Technology, Spain.

Exon skipping is one of the promising strategies to treat Duchenne Muscular Dystrophy. Recently we have studied the case of a Duchenne patient (GS $\Delta 44)$ characterized by a slower progression of the pathology and healthier conditions, if compared to other age-matched Duchenne patients. Studies in our lab revealed that his situation is due to a natural exon skipping occurring in his cells. Briefly, muscles of this subject lack the expression of CELF2a splicing enhancer. This protein is involved in splicing of exon 45, and when is absent, like in GS $\Delta 44$, this exon isn't properly recognized and, in a sub-population of Dystrophin transcripts, is skipped. This phenomenon, in a DMD $\Delta 44$ context, like the GS $\Delta 44$ one, allows the restoration of the frame of the transcript and the production of a small amount of the Dystrophin protein. Starting from this evidence, it seemed interesting to deepen the study of the regulation of this protein since its inhibition could be a treatment for those patients in which the skipping of exon 45 could recover the Dystrophin mRNA frame. At first, we investigated the reason of the absence of the protein in this individual. Genetic mutations were identified nor in the promoter neither in the coding sequence. Moreover, iPSC derived from GS $\Delta 44$ fibroblasts and differentiated to myotubes, regain the expression of CELF2a. These data suggest that an aberrant epigenetic control doesn't allow CELF2a expression in the patient. In order to elucidate this regulation, we decided to perform ChIP-seq and ATAC-seq in GS $\triangle 44$ versus control myoblasts. The first was performed to study common histones modifications, the latter to take a snapshot of the genome wide conformation of the chromatin. In this way we discriminate loci characterized by opened and actively transcribed chromatin and closed and inactive regions. CELF2a regulatory region is marked by closed chromatin and repressive histone labels in GS $\Delta 44$ (H3K27me3), while in control is open and shows histone modifications that are usually enriched at active chromatin regions (H3K4me3 and $\mathrm{H} 3 \mathrm{~K} 27 \mathrm{ac}$ ), in line with our observations. To further understand the reason of the missing expression of CELF2a in the patient, RNA-seq analysis was performed on GS $\Delta 44$ and control myoblasts, in order to find a regulator of the factor. No differences in transcription factor expression, between the two condition, were found. Instead we identified some long noncoding differentially expressed. Among 
these, one is particular interesting: some experiments correlated its presence in the patient to the absence of CELF2a. We hypothesize that this long noncoding could repress CELF2a expression through the fine-tuning of chromatin accessibility. At the same time, fibroblasts derived from a $\Delta 44$ patient were reprogramed to iPSC and clones knock out for CELF2a were obtained through CRISPr/Cas9 technique. Interestingly these clones are able to skip exon 45 thus confirming the therapeutical potential of the inhibition of CELF2a.

\subsection{New emerging role for HDAC4 on sarcolemma stability in DMD}

Alessandra Renzini, Giorgia Cavioli, Sergio Adamo, Viviana Moresi

Dept of Anatomical, Histological, Forensic \& Orthopaedic Sciences, Histology \& Medical Embryology Section, Sapienza University of Rome, Italy

Histone deacetylase 4 (HDAC4) is a member of class II HDACs which, by cooperating with class I HDACs, deacetylates proteins, thereby mediating the response to different stimuli in skeletal muscle. Recently its protective, essential role in maintaining skeletal muscle homeostasis after long-term denervation (1) or in Amyotrophic Lateral Sclerosis (2) and in satellite cell proliferation and differentiation (3) has been clarified. Further, HDAC4 is crucial for skeletal muscle regeneration by mediating soluble factors that influence musclederived cell proliferation and differentiation after injury (4). Duchenne Muscular Dystrophy (DMD) is a fatal inherited muscle-wasting disease, caused by mutations in the dystrophin gene (5) and characterized by progressive muscle weakness and degeneration. The membrane repair response, which involves multiple proteins such as dysferlin, MG53 and Annexin 1, is enhanced to actively maintain membrane integrity in DMD (6). The pan-HDAC inhibitor givinostat is presently in phase III clinical trial for the treatment of DMD, despite no efficacy in skeletal muscle function has been registered in DMD patients (7), highlighting the needs to study the HDAC functions in skeletal muscle in DMD further. While the function of class I HDACs in DMD has been partially elucidated (8), little is known about the role of class II HDACs. To shed light on additional functions of HDAC4 in skeletal muscle, we are currently studying the role of HDAC4 in DMD by using a genetic approach. We generated DMD mice with HDAC4 deleted in skeletal muscle (mdx;HDAC4mKO), by crossing mice with a skeletal muscle-specific deletion of HDAC4 (HDAC4 mKO) with mdx mice, a mouse model of DMD. To determine HDAC4 functions, muscular dystrophy progression has been analyzed over time, by histological and functional analyses. The deletion of HDAC4 in skeletal muscles exacerbates muscle degeneration, increases circulating creatine kinase levels and decreases muscle functionality over time. Further investigations have highlighted an impaired membrane repair mechanism in mdx;HDAC4mKO mice that may underpin the more pronounced progression of the pathology. From our results, we conclude that HDAC4 is important for maintaining skeletal muscle integrity in DMD mice. Ongoing studies are necessary to define the molecular signaling modulated by HDAC4 in DMD in order to provide the experimental basis for a more efficacious pharmacological therapy.

1. Pigna E. et al. 2018 Skelet Muscle. Feb 24;8(1):6.

2. Pigna E. et al. 2019 EBioMedicine. Feb;40:717-732.3.

3. Marroncelli N. et al. 2018 Sci Rep. Feb 22;8(1):3448.

4. Renzini A. et al. 2018 Front Physiol. Sep 27;9:1387.

5. Blake DJ. et al. 2002 Physiol Rev. Apr;82(2):291-329.

6. Vila MC. Et al. 2017 Cell Death and differentiation 24, 330-342.

7. Bettica P. et al. 2016 Neuromuscul Disord. Oct;26(10):643-649.

8. Colussi C. et al. 2008 Proc Natl Acad Sci U S A. Dec 9;105(49):19183-7. 


\subsection{Muscle stem cells from developing children with cerebral palsy: a pilot study}

Marlies Corvelyn ${ }^{\mathrm{a}}$, Nathalie De Beukelaer ${ }^{\mathrm{b}}$, Jorieke Deschrevelc ${ }^{\mathrm{c}}$, Anja Van Campenhout ${ }^{d}$, Sandra Prinsen ${ }^{\mathrm{d}}$, Ghislaine Gayan-Ramirezc, Kaat Desloovere ${ }^{b}$, Maurilio Sampaolesia , Domiziana Costamagna ${ }^{a}$

a. Stem Cell Biology and Embryology, Dept. of Development and Regeneration - b. Neurorehabilitation group, Dept. of Rehabilitation Sciences - c. Pneumology, Dept. of Chronic Diseases, Metabolisms and Ageing - d. Pediatric Orthopedics, Dept. of Development and Regeneration, KU Leuven, Belgium.

Cerebral palsy (CP) is the single largest condition leading to childhood physical disability. This neurodevelopmental disorder is characterized primarily by neural deficits caused by a non-progressive lesion in the immature brain, and secondly by musculoskeletal problems that progress with age. Alterations at the level of the muscle have been observed in comparison to normal developing children on both macroscopic (e.g. reduced muscle volume, shorter muscle belly, longer tendons) and microscopic (e.g. hypertrophic extracellular matrix and fibrotic tissue accumulation) levels. Unfortunately, the onset and the development of these muscle alterations are not well understood, since data on young CP patients are still lacking. The skeletal muscle is a highly dynamic tissue and resident stem cells are the postnatal actors of repair and remodelling processes. Therefore, to better understand the impaired development of the muscle and the underlying mechanisms responsible for the alterations reported in $\mathrm{CP}$ children, we aim to comprehensively describe the features of the stem cells from CP children compared to stem cells from Typically Developing children (TD). As a starting point for an extended research project on the macroscopic and microscopic muscle properties of these children, the current study reports the pilot results on the microscopic level. A minimally invasive needle microbiopsy (16G) was applied to obtain muscle biopsies from the Medial Gastrocnemius, during interventions that required general anaesthesia. A total of $10 \mathrm{CP}$ patients with Gross Motor Function Classification System level I-III were included within a range from 2 to 9 years old. Biopsies on TD children are currently ongoing. The microbiopsy technique was well-tolerated. The explant technique resulted in a reasonable number of cells for all the planned in vitro examinations. Satellite cells (SCs), mesoangioblasts and fibro-adipogenic progenitor-like cells were isolated using Fluorescence-activated cell sorting based on respectively CD56, Alkaline Phosphatase and PDGFRa protein expression. All cell types were characterised by their myogenic and/or adipogenic differentiation potential. Six-day differentiation of SCs led to heterogeneous levels of differentiation and maturation based on the expression of Myosin Heavy Chain protein and on fusion indexes. In addition, on these myotubes, disturbed nuclear migration was observed. In conclusion, microbiopsy proved to be harmless and efficient for our purposes. In ongoing research, a higher number of patients and multiple age-matched TD children are being included to confirm our preliminary observations. Future analysis will focus on nuclear migration and other impaired features that will be further assessed in the upcoming months.

\subsection{Physiopathological characterization of the role of MCUb in skeletal muscle regeneration}

S. Feno, F. Munari, A. Viola, A. Raffaello, R. Rizzuto

Dept. of Biomedical Sciences, University of Padova, Italy

Mitochondrial calcium uptake plays a key role in modulating cell metabolism, cell survival and other crucial cell functions. Calcium accumulates into the mitochondrial matrix through the mitochondrial calcium uniporter (MCU). Few years ago a MCU homolog has been discovered, that was called MCUb. MCU and MCUb share a $50 \%$ sequence and structure similarity although some conserved differences in the primary sequence prevent MCUb from forming a $\mathrm{Ca}^{2+}$-permeable channel, thus acting as a dominant-negative subunit. Interestingly, $\mathrm{MCUb} / \mathrm{MCU}$ expression ratio varies greatly among tissues, suggesting that it might contribute to the spatiotemporal control of mitochondrial calcium uptake. Here we show that MCUb is highly expressed during skeletal muscle regeneration, a process that is mainly regulated by phenotypic skewing of tissue resident and freshly recruited macrophages. We have demonstrated that MCUb expression is specifically induced in macrophages, the most abundant cell population in a regenerating muscle that drive both the proliferation and differentiation of the muscle stem cells, satellite cells, thus affecting the progression of healing after muscle damage. In vitro and in vivo experiments demonstrated that the absence of MCU affects macrophages skewing from a pro (M1) to an anti-inflammatory profile (M2). Indeed, macrophages of mice lacking MCUb are more prone to acquire a pro-inflammatory M1 profile. Since M2 macrophages have a proregenerative role and promote myogenic precursor cell differentiation, we hypothesized that MCUb absence, by affecting M2 polarization, might influence the correct remodeling and reorganization of skeletal muscle structure after damage. Our results clearly show that regenerating muscles lacking MCUb show a decrease in the expression level of myogenic regulatory transcription factors involved in satellite cells activation and differentiation. We also observed a decrease in the percentage of regenerating myofibers and a decrease in collagen deposition. In addition, co-culture experiments demonstrated that macrophage-conditioned medium 
from M2 MCUb KO macrophages drastically decreases satellite cell differentiation. Altogether these results demonstrate the role of mitochondrial $\mathrm{Ca}^{2+}$ uptake in promoting the polarization of macrophages towards an M2 pro-regenerative profile during skeletal muscle regeneration. Further experiments will help to clarify the role of mitochondrial calcium during skeletal muscle regeneration.

\subsection{Splenic Ly6C ${ }^{\text {hi }}$ monocytes are critical players in dystrophic muscle injury and repair} Giuseppe Rizzo\#, Rosanna Di Maggio, Anna Benedetti, Jacopo Morroni, Marina Bouche, Biliana LozanoskaOchser

Dept. of Anatomical, Histological, Forensic and Orthopedic Sciences, Sapienza University of Rome, Rome, Italy - \# Present address: University Hospital Würzburg, Germany; Comprehensive Heart Failure Center, Würzburg, Germany

Dystrophic muscle is characterised by chronic injury, and a steady recruitment of inflammatory Ly6Chi monocytes. Recent studies have identified the spleen as the dominant reservoir of these cells during chronic inflammation. Here we investigated the hitherto unexplored contribution of splenic Ly6 $\mathrm{C}^{\text {hi }}$ monocytes to dystrophic muscle pathology. Using the $m d x$ mouse model of muscular dystrophy, we show that Ly $6 \mathrm{C}^{\text {hi }}$ monocytes accumulate in great numbers in the spleen over the course of the disease. The chemokine receptor CCR2 was upregulated before disease onset, enabling their egress from the spleen. Splenectomy performed before disease onset significantly reduced the number of Ly6 $\mathrm{C}^{\text {hi }}$ monocytes infiltrating dystrophic limb muscle. Moreover, in the absence of splenic Ly6Chi monocytes there was a significant reduction in dystrophic muscle inflammation and necrosis, along with improved regeneration during early disease. However, during late disease, lack of splenic Ly6C ${ }^{\text {hi }}$ monocytes adversely affected muscle fiber repair, caused a delay in the phenotypic shift of pro-inflammatory $\mathrm{F} 4 / 80^{+} / \mathrm{Ly}_{6 \mathrm{C}} \mathrm{h}^{\mathrm{h}} / \mathrm{CD} 206^{\mathrm{lo}}$ to anti-inflammatory $\mathrm{F} 4 / 80^{+} / \mathrm{Ly} 6 \mathrm{C}^{\mathrm{lo}} / \mathrm{CD} 206^{+}$ macrophages, and was associated with increased fibrosis. Overall, we show that the spleen is an indispensable source of Ly6 $\mathrm{C}^{\text {hi }}$ monocytes in muscular dystrophy, and that splenic monocytes are critical players in both muscle fiber injury and repair.

\subsection{Approaches to delay the progression of Muscular Dystrophy}

Giuseppe Angelini, Giada Mura, Marielle Saclier, Valentina Taglietti, Antonini Stefania, Bonfanti Chiara, Graziella Messina

Dept. of Biosciences, University of Milan, Via Celoria 26, 20133, Milan, Italy

Muscular Dystrophies (MDs) are severe genetic disorders mainly due to mutations in structural proteins, causing contraction-induced damages. Previous attempts to treat these diseases raised from the idea that accelerating muscle growth and regeneration would exert beneficial effects. We recently demonstrated that slowing down the degeneration-regeneration cycles and switching muscle fibers towards a slow-twitching phenotype by silencing the transcription factor Nfix leads to a morphological and functional amelioration of the dystrophic phenotype. On the basis of the identification of the molecular pathways regulating Nfix expression, we are now developing a pharmacological approach to inhibit Nfix in MDs which is based on the inhibition of the ERK pathway by using two FDA- and EMA approved drugs for cancer. At the same time, another strategy considered is the use of antioxidants to protect myofibers from oxidative stress generated by muscular contraction. Different evidences are indeed establishing the importance of dietary anthocyanin's for preventive and ameliorative strategies against chronic diseases. We therefore tested the therapeutic benefit of a cyanidinenriched diet in the progression of the MD. Interestingly, dystrophic mice fed with a cyanidin-enriched diet show a morphological and functional amelioration of the dystrophic phenotype through mechanisms that involve both cell survival and anti-inflammatory pathways. All this evidence, strongly demonstrate that promising therapeutic and supporting strategies to slow down the disease progression in dystrophic patients is to reduce, the oxidative stress, muscle regeneration and muscle contraction achievable through different and synergistic strategies.

2.5 Dissecting the secretome during muscle regeneration: role and kinetics of cytokines and extracellular vesicles.

Simone Vumbaca, Claudia Fuoco, Andrea Cerquone Perpetuini, Valeria Fiorentini, Giulio Giuliani, Luisa Castagnoli, Gianni Cesareni

Dept. of Biology, University of Rome "Tor Vergata", Italy

Muscle regeneration is a coordinated process that ultimately results in the recovery of tissue functionality after damage. Half a dozen cell types work in unison and create a complex communication network that changes in space and time. The dynamics and kinetics of this network are determined by the secretion of cytokines, extra-cellular vesicles (EVs) and extra-cellular matrix (ECM). For the purpose of dissecting the secretome, we 
profiled both the cytokines and the EVs at different time points during muscle regeneration of the cardiotoxin injured mouse model. We performed a multiplex ELISA assay of the mononuclear cell compartment lysate to analyze the expression kinetics of 90 cytokines. In addition, by profiling the RNA expression profile of four cell populations, we determined the cell types that have the potential to secrete the different cytokines and those that synthetize the specific receptors. Based on the kinetic of their expression during damage recovery, we selected 15 cytokines to be tested in vitro on fibro-adipogenic progenitors (FAPs). Using this approach we identified cytokines that influence FAP proliferation and differentiation decisions. In addition, by culturing injured muscles ex-vivo, we obtained the EVs secreted by the tissue during the regeneration process. To analyze their cargo, we characterized the vesicle heterogeneity by using a flow cytometry approach. Specifically, we identified and quantified the RNAs- and enzymes-containing EVs. Finally, we investigated their ability to interact with different muscle-derived cell populations, influencing their proliferation and differentiation potential. Our data, combined with in silico modeling, will increase our understanding of cell-cell communication during muscle regeneration. 
3.1 Organ-on-a-chip modelling of the human neuromuscular junction in both physiological and pathological conditions.

Ersilia Fornettia , Stefano Testa ${ }^{a}$, Victorio Pozo-Devoto ${ }^{b}$, Alberto Rainer ${ }^{c}$, Giancarlo Forte ${ }^{b}$, Cesare Gargiolia, Stefano Cannata ${ }^{\mathrm{a}}$

a. Dept. of Biology, University "Tor Vergata”, Rome - b. Center for Translational Medicine, FNUSA ICRC, Brno, Czech Republic - c. Tissue Engineering Unit, University "Campus Bio-Medico", Rome, Italy.

The neuromuscular junction (NMJ) is a chemical synapse localized between the terminal branches of the spinal motor neurons and the skeletal muscle fibers. In the past two decades co-culture systems to recreate the NMJ in vitro were developed to address concerns about animal models, nevertheless the complexity of its highly specialized structure makes the in vitro modeling a challenging task. However, a further improvement in this issue was recently provided by microfluidics that, unlike in mass co-cultures, allow spatial and temporal control over different microenvironment by manipulating either neural cells or muscle cells populations independently. This enables the investigation of mechanisms involved in the formation and the maintenance of a stable and functional NMJ. Therefore, exploiting an organ-on-a-chip approach, our aim is to obtain a reliable and predictive in vitro human model of $\mathrm{NMJ}$ in physiological and pathological conditions, to investigate the occurrence of synapse detriment in neuromuscular diseases. For this purpose, motor neurons derived from human iPSCs and human skeletal muscle cells derived from either perivascular muscle progenitors, namely Pericytes, or immortalized myoblasts are seeded in two separated chambers of a microfluidic device. The two side of the device are linked together through microchannels that enable the axonal outgrowth to the muscle side, but not cell bodies migration, allowing the compartmentalization of the cell populations without interrupting cell-cell communication. While being designed as a reliable platform to investigate the molecular actors of NMJ processes, the setup is versatile enough to host patient-specific cells and perform functional and molecular analysis.

\subsection{Polyglutamine-expanded androgen receptor alters excitation-contraction coupling machinery and calcium dynamics}

Caterina Marchioretti ${ }^{1,2}$, Mathilde Chivet $^{3}$, Marco Pirazzini ${ }^{1}$, Diana Piol1,2, Maria Polancoㄹ, Vanina Romanello, $2,3,4$, Maurizio D'Antonio ${ }^{6}$, Fabio Sambataro7,8, Elena Pegoraro7,8, Gianni Sorarù7,8, Marco Sandri2,3,4, Manuela Basso ${ }^{9}$, Maria Pennuto $1,2,3,4,5,8$

1. Dept. of Biomedical Sciences (DBS), University of Padova -2. Veneto Institute of Molecular Medicine (VIMM) - 3. Dulbecco Telethon Institute, Centre for Integrative Biology (CIBIO), University of Trento - 4. Myology Center (Cir-Myo), University of Padova - 5. Dept. of Neuroscience and Brain Technologies, Istituto Italiano di Tecnologia (IIT), Genova - 6. Division of Genetics and Cell Biology, San Raffaele Scientific Institute, Milan - 7. Dept. of Neuroscience (DNS), University of Padova - 8. Padova Neuroscience Center (PNC) - 9. Centre for Integrative Biology (CIBIO), University of Trento, Italy

Spinal and bulbar muscular atrophy (SBMA) is caused by polyglutamine (polyQ) expansions in the AR gene. Although clinical and experimental evidence highlight a primary role for skeletal muscle in the onset, progression, and outcome of SBMA, the pathophysiological and molecular processes underlying SBMA muscle atrophy are poorly understood. We show that polyQ-expanded AR alters intrinsic muscle force generation before denervation. Reduced muscle force was associated with a switch in fiber-type composition, disrupted muscle striation, altered calcium $(\mathrm{Ca}++)$ dynamics in response to muscle contraction, and aberrant expression of excitation-contraction coupling (ECC) machinery genes in transgenic, knock-in and inducible SBMA mice and patients. Acute suppression of AR activation by surgical castration elicited similar ECC gene expression changes in normal mice, suggesting that AR regulates the expression of these genes in physiological conditions. Importantly, treatment to suppress polyQ-expanded AR expression restored ECC gene expression back to normal. Bioinformatic analysis revealed the presence of androgen-responsive elements on several genes involved in muscle function and homeostasis. Experimental evidence showed ARdependent regulation of expression and promoter occupancy of the most up-regulated gene from transcriptomic analysis in SBMA muscle. These observations reveal an unpredicted role for AR in the regulation of expression of genes involved in muscle contraction and $\mathrm{Ca++}$ dynamics, a level of muscle function regulation that is disrupted in SBMA muscle, yet restored by pharmacologic treatment.

\subsection{Transverse Tubule Remodeling Enhances Orai1-dependent $\mathrm{Ca}^{2+}$ Entry in Skeletal Muscle Antonio Michelucci ${ }^{1,2}$, Simona Boncompagni ${ }^{2}$, Laura Pietrangelo², Robert T. Dirksen ${ }^{*}$, Feliciano Protasi2 ${ }^{*}$. Dept. of Pharmacology and Physiology, University of Rochester School of Medicine and Dentistry, Rochester, NY 14642 - 2. CeSI-MeT, Center for Research on Ageing and Translational Medicine; University G. d' Annunzio of Chieti, Italy - * Equally contributing authors In skeletal muscle, acute exercise promotes the formation of intracellular junctions between stacks of sarcoplasmic reticulum (SR) cisternae (SR-stacks) and transverse-tubule (TTs) extensions. These junctions}


(referred to as $\mathrm{Ca}^{2+}$ entry units or CEUs) promote increased co-localization of Stim1 $\mathrm{SR} \mathrm{Ca}^{2+}$ sensors and $\mathrm{Ca}^{2+}$-permeable Orai1 channels, the core machinery of store-operated $\mathrm{Ca}^{2+}$ entry (SOCE). However, CEU stability following exercise and the impact of their formation/disassembly on SOCE, Ca ${ }^{2+}$ dynamics, and muscle contractility are unknown. Here, we addressed these issues using a combination of electron microscopy, $\mathrm{Mn}^{2+}$ quench, $\mathrm{Ca}^{2+}$ dynamics, and muscle contractility measurements in extensor digitorum longus muscle and flexor digitorum brevis fibers from mice $<1,6$, and 24 hours after acute treadmill exercise. As shown previously, SR-stacks with associated TT extensions increased $<1$ hour after exercise. While SRstacks number/area increased further at 6 hours and returned to control levels 24 hours after exercise, TTs extensions were retracted from SR-stacks prior to 6 hours after exercise. SOCE activity following store depletion, as well as peak $\mathrm{Ca}^{2+}$ transient amplitude and contractile force during repetitive stimulation increased $<1$ hour after exercise, but returned to control levels at 6 and 24 hours. Unexpectedly, constitutive SOCE coincident with modest store depletion and increased resting $\mathrm{Ca}^{2+}$ were observed only $<1$ hour after exercise. The functional benefits of acute exercise on SOCE activity and muscle contractility were lost in mice lacking Orai1 function in muscle. These results suggest that TT association with SR-stacks enhances Orai1dependent SOCE to optimize $\mathrm{Ca}^{2+}$ dynamics and muscle contractile function during acute exercise. 


\begin{abstract}
4.1 The regulation of protein synthesis and muscle plasticity
Ana Georgia Dumitras ${ }^{\mathrm{a}}$, Leonardo Nogara a,b, Marcus Krugerc, Bert Blaauw a,b

a. Venetian Institute of Molecular Medicine, Padua, Italy - b. Dept. of biomedical sciences, Univ. of Padua, Padua, Italy - c. CECAD Research Center, University of Cologne, Germany

The aim of this project is to dissect the identity and localization of the nascent muscle proteome in vivo. The tool of choice is the transgenic mouse MetRS, expressing a mutant methionyl-tRNA synthetase together with a green fluorescent protein. We crossed this mouse with a skeletal muscle specific CRE-expressing mouse to obtain a tissue specific expression. The mutated methionyl-tRNA synthetase can integrate a synthetic amino acid (ANL) instead of a methionine in a nascent peptide chain. The synthetic amino acid side chain ends with an azide, allowing the formation of a covalent bond with an alkyne by a click chemistry reaction. To visualize the GFP-2A-MetRS expression, a western-blot with anti GFP antibody has been performed as well as immunohistochemistry on cryosections. The flexibility of the click reaction allows the visualization of nascent peptide chains either by western blot or immunofluorescence. Intraperitoneal injections of ANL for different time periods resulted in a significant labelling of the muscle proteome after 1,2 and 7 days. After the protein extraction, each whole cell lysate has been clicked with biotin labelled alkyne in the presence of copper (II) sulphate and ascorbic acid and labelled proteins were revealed by western blotting. Labelled proteins can also be revealed by IHC performing FUNCAT (Fluorescent non-canonical amino-acid tagging) on cryosections. This way we were able to identify that a specific proteome was synthesized during a determined amount of time. These preliminary studies will give us the necessary tools to better understand the skeletal muscle proteome in physiological and pathological conditions.
\end{abstract}

4.2 Drp1 promotes mitochondrial transport and repositioning in muscle by enhancing kinesin-1 activation

Matteo Giovarellia , Silvia Zecchinia, Dario Parazzolib, Emilio Clementia,c, Clara De Palmad

a. Dept. of Biomedical and Clinical Sciences "Luigi Sacco", Università di Milano - b. IFOM, Fondazione Istituto FIRC (Fondazione Italiana per la Ricerca sul Cancro) di Oncologia Molecolare, Milan - c. IRCCS Eugenio Medea, Bosisio Parini, Italy - d. Unit of Clinical Pharmacology, University Hospital "Luigi Sacco"-ASST Fatebenefratelli Sacco; National Research Council-Institute of Neuroscience; Dept. of Biomedical and Clinical Sciences, Università di Milano.

Mitochondria change distribution across cells following a variety of pathophysiological stimuli. The mechanisms presiding over this redistribution are yet undefined. In a murine model overexpressing Drp1 specifically in skeletal muscle, we find marked mitochondria repositioning in muscle fibres and we demonstrate that Drp1 is involved in this process. Drp1 binds KLC1 and enhances microtubule-dependent transport of mitochondria. Drp1-KLC1 coupling triggers the displacement of KIF5B from kinesin-1 complex increasing its binding to microtubule tracks and mitochondrial transport. High levels of Drp1 exacerbate this mechanism leading to the repositioning of mitochondria closer to nuclei. The reduction of Drp1 levels decreases kinesin-1 activation and induces the partial recovery of mitochondrial distribution, thus linking mitochondrial movements to Drp1 levels. This aberrant positioning of mitochondria is accompanied by the collapse and aggregation of desmin confirming that the loss of mitochondria anchoring system may contribute to mitochondria distribution. We conclude that desmin alterations accompanied by increased Drp1 levels enhance microtubule-dependent trafficking promoting changes in mitochondrial positioning unravelling a hitherto unknown function of Drp1 in the regulation of kinesin-1 complex.

4.3 The NF-Y regulome in mouse fetal myoblasts reveals a role of SREBP in differentiation. Valentina Taglietti, Diletta Dolfini, Debora Libetti, Mirko Ronzio, Graziella Messina, Roberto Mantovani Dipartimento di Bioscienze, Università degli Studi di Milano, Milano, Italy.

The "ubiquitous" CCAAT-binding transcription factor NF-Y is a trimer formed by the histone-like NF-YB/NF-YC and the sequence-specific NF-YA, present in two isoforms. All three subunits are required for DNA-binding and function. The role of NF-Y in muscle physiology is not clear: if overexpressed, the "long" NF-YA isoform favors differentiation, yet it is negatively regulated during the process. We targeted NF-YA Exon 3 by CRISPRCas9 in mouse C2C12, thus ablating the "long" NF-YA: despite expression of NF-YA "short", differentiation is blocked. To understand the NF-Y regulome in a more physiological system, we turned to primary mouse fetal myoblasts, performing ChIP-Seq, RNA-Seq after NF-YB RNAi, and monitoring gene expression from myoblasts to myotubes. NF-Y locations are similar before and after differentiation, despite far lower levels of NF-YA. Notably, the NF-Y regulome is devoid of binding sites of MRFs -Muscle Regulatory Factors- and myotubes-specific genes are not targeted. Instead, we observe a positive functional partnership with SREBPs, master regulators of lipids biosynthesis, and a negative one on cell-cycle genes. SREBP-2 is active in differentiation and bound to promoters of genes co-regulated with NF-Y. These data establish that NF-Y, and 
specifically the "long" NF-YA isoform, is essential for myotubes formation, but marginally, if at all, through direct activation of muscle-specific genes. Instead, it teams up with SREBPs to activate crucial steps of lipids metabolic pathways required for the process. The NF-Y/SREBPs partnership will be discussed.

\subsection{Unveiling novel and specific functions of protein kinase CK2 subunits in skeletal muscle differentiation and fusogenic activity \\ Sofia Zanin ${ }^{a}$, Valentina Salizzato ${ }^{a}$, Valentina Salizzato ${ }^{a}$, Christian Borgo ${ }^{a}$, Rosario Rizzuto ${ }^{a}$, Arianna Donella- Deana ${ }^{a}$, Giorgia Pallafacchina ${ }^{a, b}$ \\ a. Dept. of Biomedical Sciences, University of Padua - b. Neuroscience Institute, CNR National Research Council, Italy \\ CK2 is a tetrameric protein-kinase, composed of two catalytic ( $\alpha$ and/or $\left.\alpha^{\prime}\right)$ and two regulatory $\beta$-subunits. Our study provides the first molecular and cellular characterization of the different CK2-subunits highlighting their individual roles in skeletal muscle specification and differentiation.}

Analysis of $\mathrm{C} 2 \mathrm{C} 12$ cells knockout for each CK2-subunit reveals that: i) CK2 $\beta$ is mandatory for the expression of the muscle master-regulator MyoD in proliferating myoblasts, thus controlling both myogenic commitment and subsequent muscle-specific gene expression and myotube formation; ii) CK2 $\alpha$ is involved in the activation of the muscle-specific gene-program; iii) CK2 $\alpha$-activity regulates myoblast fusion by mediating plasma membrane translocation of fusogenic proteins essential for membrane coalescence, like myomixer. Accordingly, CK2 $\alpha$ ' overexpression in $\mathrm{C} 2 \mathrm{C} 12$ cells and in mouse regenerating muscle is sufficient to increase myofiber size and myonuclei content via enhanced satellite cell fusion. Consistent with these results, pharmacological inhibition of CK2-activity substantially blocks the expression of myogenic markers and muscle cell fusion both in vitro, in $\mathrm{C} 2 \mathrm{C} 12$ and primary myoblasts, and in vivo, in mouse regenerating muscle and zebrafish development. Concluding, our work describes the specific and coordinated functions of CK2-subunits in orchestrating muscle differentiation and fusogenic activity, highlighting CK2 relevance in the physiopathology of skeletal muscle tissue.

4.5 Adipogenesis of skeletal muscle Fibro/Adipogenic Progenitors is controlled by the Wnt5a/GSK3/ $\square$ catenin axis

Alessio Reggio ${ }^{1,4}$, Marco Rosina ${ }^{1,4}$, Alessandro Palma ${ }^{1,4}$, Andrea Cerquone Perpetuini ${ }^{1}$, Giulio Giuliani ${ }^{1}$, Elisa Micarelli ${ }^{1}$, Lucia Lisa Petrilli' ${ }^{1}$, Cesare Gargioli ${ }^{1}$, Claudia Fuoco ${ }^{1}$, Mauro Cerretani, Alberto Bresciani, Francesca Sacco ${ }^{1}$, Luisa Castagnoli ${ }^{1}$, Gianni Cesareni ${ }^{1,2}$

1. Dept. of Biology, University of Rome "Tor Vergata" - 2. Fondazione Santa Lucia Istituto di Ricovero e Cura a Carattere Scientifico (IRCCS), Rome - 3. Dept. of Biology, IRBM S.p.A, Pomezia (Rome), Italy. - 4. Equally contributing authors.

Fibro/adipogenic progenitors (FAPs) are muscle interstitial progenitors mediating pro-myogenic signals that are critical in muscle homeostasis and regeneration. In pathological conditions, including ageing and myopathies, the autocrine/paracrine constraints controlling FAP adipogenic potential are released causing the formation of fat infiltrates. To reveal pathways that transduce the niche messages controlling FAP adipogenesis, we have screened a library of inhibitors targeting a large fraction of the human/murine kinome, looking for molecules with a potential to modulate FAP differentiation. Hit selection criteria enrich compounds targeting Glycogen Synthase Kinase 3 (GSK3), thus unveiling its crucial role in FAP adipogenesis. Consistently, we show that the pharmacological blockade of GSK3, by using the high-selective inhibitor LY2090314, severely impedes FAP adipogenesis ex vivo while limiting intramuscular fat infiltrations upon glycerol-induced muscle injury, in vivo. By exploiting high-dimensional single-cell mass cytometry, we demonstrate that $\square$-catenin down-regulation is critical for PPAR $\square$ expression. LY2090314, by targeting GSK3, counteracts this process and prevents FAP adipogenesis. By combining computational strategies based on the interrogation of single-cell transcriptomes, in silico network modeling and RNAseq data integration, we conclude that: i) FAPs are the main source of Wnt ligands in the muscle and that the release of these cytokines may trigger autocrine/paracrine responses; ii) the Wnt pathway has a dominant role in regulating the adipogenic network of FAPs; iii) Wnt5a expression is impaired in FAPs from dystrophic mice. Finally, we show that exogenous treatment of dystrophic FAPs with Wnt5a is sufficient to repress PPAR $\square$ expression and the adipogenic conversion of FAPs into adipocytes. These results suggest that by modulating the Wnt pathway either by targeting GSK3 or by restoring autocrine Wnt5a signaling in FAPs might be a strategy to counteract intramuscular fat infiltrations in myopathies. 


\subsection{Evo-Devo approach to study Pax3/7 functions}

Valentina Taglietti ${ }^{\mathrm{a}}$, Shinichiro Hayashi ${ }^{\mathrm{b}}$, Frederic Aurade ${ }^{\mathrm{a}}$, Frederic Relaix ${ }^{\mathrm{a}}$

a. Paris Est-Creteil University, IMRB U955, Faculté de médecine 8 rue du Général Sarrail, 94000 Créteil, France - b. Tokyo Medical and Dental University, Yushima, Bunkyo-ku, Tokyo, Japan

Pax3 and Pax7 are paired-homeobox transcription factors regulating progenitor and stem cells of several tissues. To analyse Pax3/7 conservation during evolution, we have replaced Pax3 by Amphioxus ancestral Pax3/7 gene. Amphioxus do not form limbs, lack migratory neural crest cells and possess only a single Pax $3 / 7$ gene. Our results show that Amphioxus Pax3/7, similar to mouse Pax7, can compensate for Pax3 deficiency in dorsal neural tube, and somite development. Surprisingly, muscle progenitor cell migration and neural crest cell migration are also restored. Our results have implications for the study of the gene regulatory network of somites and crest cells during evolution. 


\subsection{Mitochondrial adaptation in parvalbumin knockout muscle fibers}

\section{Gaia Butera, Marta Canato, Denis Vecellio Reane, Rosario Rizzuto, Carlo Reggiani, Anna Raffaello}

Dept. of Biomedical Sciences, University of Padova, Padua, Italy

In skeletal muscle, mitochondrial $\mathrm{Ca}^{2+}$ uptake plays important roles in organ homeostasis, ranging from control of metabolism to regulation of fiber trophism. Still debated is whether in muscle mitochondria can buffer cytosolic $\mathrm{Ca}^{2+}$ increases. To answer to this question, we explored the effect of removing parvalbumin (PV), the most important muscle cytosolic $\mathrm{Ca}^{2+}$ buffer. By using a PV knockout (KO) mouse model, we are investigating whether the absence of PV induces compensatory mechanisms on the expression and function of the mitochondrial $\mathrm{Ca}^{2+}$ uptake machinery (MCU). The data obtained so far confirm that the absence of PV induces an increase of mitochondrial $\mathrm{Ca}^{2+}$ uptake and this is accompanied by the induction of the expression of the MCU coin vitromplex components. Moreover, electron microscopy analysis performed on extensor digitorum longus (EDL) muscle of WT and PV KO mice, demonstrated that PV KO mitochondria are significantly larger than WT controls and with a different redistribution in the muscular tissue, suggesting a strict connection and regulation between PV expression and mitochondrial morphology and function in muscle cells. Furthermore, cytosolic $\mathrm{Ca}^{2+}$ transients in PV KO muscle fibers show that the time to reach the peak upon stimulation and the time to half relaxation are prolonged in PV KO muscles. To verify whether the increase of mitochondrial $\mathrm{Ca}^{2+}$ uptake in $\mathrm{KO}$ fibers is due to mitochondrial adaptation and whether $\mathrm{MCU}$ is responsible of buffering cytosolic $\mathrm{Ca}^{2+}$ increase, we decided to silencing MCU and measure $\left[\mathrm{Ca}^{2+}\right]$ cyt on FDB muscles of WT and PV $\mathrm{KO}$ animals. $\left[\mathrm{Ca}^{2+}\right]_{\mathrm{cyt}}$ was almost unaffected by the absence of MCU in WT animals while, in PV KO fibers, $\left[\mathrm{Ca}^{2+}\right]_{\text {cyt }}$ was significantly higher upon stimulation. Furthermore, since PV is one of the most downregulated atrogenes, the genes commonly up- and down-regulated during several types of atrophy, and that mitochondrial $\mathrm{Ca}^{2+}$ controls skeletal muscle trophism, we decided to study the role of PV on muscle mass through denervation experiments. When PV is absent, loss of muscle mass is reduced compared to WT fibers, demonstrating that PV can partially protect muscles from denervation-induced atrophy. Overall, our results indicate that $\mathrm{PV}$ plays an important role in spatiotemporal control of cytosolic $\mathrm{Ca}^{2+}$ responses on mitochondrial $\mathrm{Ca}^{2+}$ uptake and have a profound impact on skeletal muscle trophism.

\subsection{Regulation of oxidative metabolism and substrate preferences in aged skeletal muscle by mitochondrial calcium uptake \\ Gaia Gherardi, Rosario Rizzuto, Cristina Mammucari \\ Dept. of Biomedical Sciences, University of Padova, Italy}

The second messenger $\mathrm{Ca}^{2+}$ regulates a broad repertoire of cellular processes. Upon physiological stimuli, skeletal muscle mitochondria rapidly and efficiently accumulate $\mathrm{Ca}^{2+}$ into their matrix via an electrogenic pathway, that relies on the driving force of a steep electrochemical gradient. A large $\left[\mathrm{Ca}^{2+}\right]_{\mathrm{mt}}$ peak occurs dynamically in parallel to agonist-induced $\left[\mathrm{Ca}^{2+}\right]_{\text {cyt }}$ increases, thanks to the activity of the Mitochondrial Calcium Uniporter (MCU), the highly selective channel responsible for mitochondrial $\mathrm{Ca}^{2+}$ accumulation. MCU positively regulates myofiber size in physiological conditions, and counteracts pathological loss of muscle mass. We have previously demonstrated that skeletal muscle-specific $\mathrm{MCU}$ deletion (MCU ${ }^{-/}$) inhibits myofiber mitochondrial $\mathrm{Ca}^{2+}$ uptake, impairs muscle force and exercise performance. Mitochondrial $\mathrm{Ca}^{2+}$ uptake is required for effective glucose oxidation and efficient mitochondrial activity. Nonetheless in $\mathrm{MCU}^{-1}$ myofibres, impaired oxidative capacity is partially sustained by increased fatty acid (FA) oxidation. The main trigger of this metabolic rewiring is the decreased pyruvate dehydrogenase activity which is tightly controlled by mitochondrial $\mathrm{Ca}^{2+}$ accumulation. Here, we have investigated the role of mitochondrial $\mathrm{Ca}^{2+}$ uptake during skeletal muscle aging. In detail, we show that mitochondrial $\mathrm{Ca}^{2+}$ accumulation is decreased in 24 months old mice and this condition is accompanied by a decreased pyruvate dehydrogenase activity. In this scenario we demonstrate a rewiring of skeletal muscle metabolism where mitochondrial activity is sustained by FA oxidation rather than glucose. Further studies are needed to evaluate whether the restore of glucose as the main fuel for oxidative metabolism will be sufficient to counteracting aging process, including force and performance, in skeletal muscles. 


\begin{abstract}
5.3 Metabolic reprogramming of Fibro/Adipogenic Progenitors facilitates muscle regeneration Marco Rosina ${ }^{\dagger a}$, Alessio Reggio ${ }^{\dagger a}$, Natalie Krahmerd, Lucia Lisa Petrillia, Giuliano Maiolatesia, Giorgia Massaccia $^{a}$ Illari Salvatorib, Cristiana Valle ${ }^{b, c}$, Claudia Fuoco ${ }^{a}$, Alessandro Palma ${ }^{a}$, Luisa Castagnolia, Gianni Cesarenia,b, Francesca Sacco ${ }^{\mathrm{a}}$

a. Dept. of Biology, University of Rome Tor Vergata - b. Fondazione Santa Lucia Istituto di Ricovero e Cura a Carattere Scientifico (IRCCS) - c. Institute of Translational Pharmacology, CNR, Italy - d. Dept. Proteomics and Signal Transduction, Max-Planck Institute of Biochemistry, Germany - †. Equally contributing authors

Dystrophin deficiency causes chronic wasting of the skeletal muscle tissue leading to patient respiratory or heart failure and, finally, death. In addition to fiber fragility, the absence of the dystrophin protein, as in Duchenne Muscular Dystrophy (DMD), causes a variety of poorly understood secondary effects. Notably muscle fibers of dystrophic individuals are characterized by mitochondrial dysfunctions, as revealed by a reduced ATP production rate and by defective oxidative phosphorylation (OxPhos). We recently discovered that in a mouse model of DMD (mdx), the interstitial Fibro/Adipogenic Progenitor (FAP) cells are also characterized by a dysfunctional mitochondrial metabolism which correlates with an increased adipogenic differentiation potential. Using high-sensitivity mass spectrometry-based proteomics, we highlighted that a short-term high-fat diet regimen reprograms dystrophic FAP metabolism in vivo. By combining our proteomic dataset with a literature-derived signaling network, we discovered a high-fat dependent modulation of the crucial hub protein, $\beta$-catenin, which controls follistatin expression. Our results reveal that a short-term highfat diet restores the key role of FAPs in enhancing the myogenic activity of the skeletal muscle stem cells in dystrophic mice. Consistently, we observe that muscle regeneration in the mdx mouse is significantly improved by the short-term high-fat diet. Our results support metabolic reprogramming of muscle interstitial progenitor cells as a novel approach to alleviate some of the adverse outcomes of Duchenne Muscular Dystrophy.
\end{abstract}

\title{
5.4 Identification of a novel TFEB and exercise dependent gene
}

Davide Steffan ${ }^{\mathrm{a}, \mathrm{b}}$, Andrea Armani ${ }^{\mathrm{a}, \mathrm{b}}$, Marco Sandria,b

a. Dept. of Biomedical Sciences, University of Padua - b. Veneto Institute of Molecular Medicine, Padua, Italy

If we concentrate Homo sapiens existence in just 24 hours, the time he started to be totally sedentary is just 30 seconds ago, while his life expectation exceeded the 60 -year during the last 25 seconds. The combination of a novel life-style with an increased life-span, two conditions that our species never experienced before, resulted in the onset of many chronic and metabolic diseases. Nevertheless, emerging evidence shows that readopting a "more active" life-style is associated with healthier and longer life-span. Whole-body beneficial effects of physical activity are the consequence of adaptive metabolic changes established in the entire organism to sustain the high energy expenditure of contracting skeletal muscles. During last years, many efforts have been made to dissect the molecular mechanisms explaining why physical activity sustains and improves life quality. Recently, our group identified the Trascription-Factor EB as a muscular master metabolic regulator that translocates into myonuclei in response to exercise promoting the expression of genes for different fuel disposal and utilization. These findings strongly support TFEB activity as crucial for the beneficial effects of exercise. Starting from this evidence we decided to discover new uncharacterized genes that may mechanistically explain adaptive effects of TFEB on skeletal muscle. By crossing gene expression data of TFEB transgenic and trained muscles, we found a gene of unknown function belonging to the Riken cDNA collection that we called Exe-Riken.Exe-Riken encodes a putative 125 residues protein highly conserved among mammals; interestingly, Exe-Riken coding sequence translates in a real protein when expressed in vitro and in vivo. Based on gene expression databases, brown adipose and gut are the tissues with higher basal Exe-Riken expression in mice. On the other hand, skeletal muscle transcript levels are very low, type of muscle specific and finely tuned with different exercise protocol. In addition, Exe-Riken protein sequence presents a Nuclear Export Signal (NES) and Nuclear Localization Signal (NLS), suggesting a potential shuttling behavior upon appropriate stimuli. Supporting this idea, in vivo experiments demonstrate Exe-Riken nuclear translocation upon exercise. Finally, overexpression studies in adult Tibialis Anterior muscle showed an increased SDH activity and a decreased PAS staining, suggesting its impact in muscle metabolism. Overall these findings highlight a possible role of this new gene in controlling metabolic adaptations during physical activity. 


\subsection{Direct effects of vitamin $D_{3}$ (cholecalciferol) on skeletal muscle}

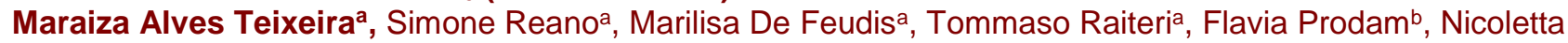

Filigheddua

a. Dept. of Translational Medicine - b. Dept. of Health Sciences, University of Piemonte Orientale, Novara, Italy

Cholecalciferol, or vitamin $\mathrm{D}_{3}$ (VD hereafter for brevity), besides its well-known role in regulation of calcium and phosphate homeostasis, impacts on skeletal muscle homeostasis as well, and VD deficiency has been correlated with decrease in muscle mass, function, and performance in elderly subjects, as well as in cachectic cancer patients. While VD supplementation was able to restore muscle strength and prevent muscle mass loss in frail elderly subjects, it was ineffective in the case of cancer cachexia-associated muscle wasting. Investigating the direct effects of the main cholecalciferol-derived metabolites -namely 1,25-(OH) $2-\mathrm{VD}$ and its precursor 25-OH-VD- on skeletal muscle cells, we found divergent effects of these molecules in promoting/preserving from atrophy induced by pro-cachectic cytokines. Given the anti-atrophic action of 25$\mathrm{OH}-\mathrm{VD}$, we wondered if also the pro-hormone VD could have a direct activity on skeletal muscle cells. For this purpose, we used an in vitro model of cytokine-induced atrophy and found that also VD has a protective action. Intriguingly, the activity of VD is notmediated by an intracellular conversion in the protective form $25-\mathrm{OH}-\mathrm{VD}$.

\section{2 rGDF5, an unexpected treatment against age-related muscle mass loss}

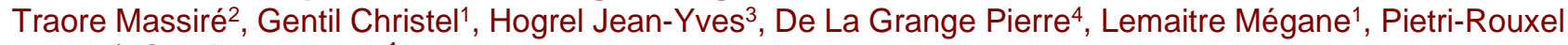
France $^{1}$, Sestina Falcone ${ }^{1}$

1. Sorbonne Université, Centre de Recherche en Myologie, INSERM U974 Institut de Myologie - 2. Inovarion - 3. Institut de Myologie, GH Pitié-Salpêtrière - 4. Genosplice, Paris, France

Our recent study revealed the existence of a novel embryonic isoform of CaV $\beta 1$ (CaV $\beta 1 \mathrm{E})$, the beta subunit of CaV1.1, which expression increases in adult muscle after denervation. The increase of CaV $\beta 1 \mathrm{E}$ expression boosts downstream Gdf5 signaling to counteract muscle atrophy due to nerve damage. We further reported that aged muscle expresses significantly reduced levels of CaV $\beta 1 E$ and that its overexpression improves mass wasting in aging muscle by increasing Gdf5 expression. Crucially, we also identified the human CaV $\beta 1 \mathrm{E}$ analogous and showed a correlation between the decrease of $\mathrm{CaV} \beta 1 \mathrm{E}$ expression and the age-related muscle mass decline in people. Altogether, our data open a new field in the development of strategies against muscle mass loss during senescence. Here, we have preliminary data indicating that the systemic administration of the recombinant Gdf5 protein (rGdf5) represents a promising therapeutic approach to improve age-related muscle wasting. In a pilot study, old mice (90 weeks old) were treated with rGdf5, by systemic delivery, for ten weeks. Strikingly, rGdf5 implementation induced a significant increase of lean mass and a decrease of the fat mass. Indeed, muscle/body-weight ratios of TAs and Quadriceps (Quad) were significantly increased in the rGdf5- 100 weeks old mice compared to vehicle-treated old mice. In order to evaluate the possible mechanisms behind rGdf5 effects in muscle, we analyzed: a) Myostatin (Gdf8) pathway, involved in protein degradation; b) neuromuscular junction (NMJ) status by measuring the expression of acetylcholin receptor subunit epsilon (Chrne). We found a significant decrease in myostatin pathway activation in muscle treated with rGdf5, suggesting his protective role against myostatin-dependent protein degradation. Furthermore, while Chrne transcription was significantly decreased during aging revealing an alteration of NMJ, rGdf5 administration led to Chrne re-expression suggesting an improvement of NMJ state. All together these data show that rGdf5 implementation efficiently counteracts age-related muscle wasting and, more generally, could ameliorate neuromuscular decline and protein degradation underlying several (neuro) muscular diseases. 


\subsection{The role of vitamin D binding protein in the onset of cancer cachexia - beyond vitamin D transport} Simone Reano ${ }^{1}$, Andrea Scircoli ${ }^{1}$, Tommaso Raiteri ${ }^{1}$, Ivan Zaggia ${ }^{1}$, Marilisa De Feudis ${ }^{1}$, Maraiza Alves Teixeira ${ }^{1}$, Emanuela Agosti ${ }^{1}$, Flavia Prodam² ${ }^{2}$, Nicoletta Filigheddu ${ }^{1}$

1. Dept. of Translational Medicine - 2. Dept. of Health Sciences, University of Piemonte Orientale, Novara, Italy

Cachexia is a highly debilitating multifactorial syndrome associated with various diseases and is characterized by severe muscle wasting leading to pronounced weight loss, impaired quality of life, reduced response to therapy, and premature death. Vitamin D binding protein (VDBP), also known as Group-specific component (Gc-globulin), is a multifunctional serum glycoprotein synthesized by hepatocytes that belongs to the albumin gene family. Besides the binding and transport of vitamin D metabolites in blood, VDBP has other activities, including binding and clearance of monomeric G-actin released from dead cells and acting as a chemotactic cofactor for C5a.Proteomic analysis of samples from patients affected by pathologies susceptible to progressive muscle loss or cachexia, including several types of cancers, shows upregulation of vitamin $D$ binding protein (VDBP), raising the hypothesis that VDBP might play a role in cancer cachexia-associated muscle loss. We found that VDBP expression increases in tumor-bearing mice undergoing cachexia, further supporting the notion that VDBP could play a role in the onset/progression of cancer-associated muscle wasting. We explored the direct action of VDBP in skeletal muscle and found that VDBP indeed induces atrophy in $\mathrm{C} 2 \mathrm{C} 12$ myotubes and that mice devoid of VDBP are protected from cancer-associated cachexia. Our preliminary results demonstrate that VDBP is not a simple carrier that modulates the bioavailability of vitamin $\mathrm{D}$ but an active hormone in itself with pro-cachectic effects on skeletal muscle

\subsection{A metabolic shift drives cancer cachexia in myotubes}

Michele Mannelli, Tania Gamberi, Francesca Magherini, Tania Fiaschi

Dept. of Experimental and Clinical Biomedical Sciences "Mario Serio", University of Florence, Italy

Cancer cachexia is a devasting multifactorial syndrome characterized by the progressive unintentional body weight loss mainly due to skeletal muscle wasting and atrophy that occurs in the majority of terminally-ill cancer patients. Notably, cancer-induced muscle wasting markedly results in a drastic worsening of patient prognosis and quality of life. Furthermore, cancer-driven cachexia also limits the therapeutic options, as cachectic patients usually manifest reduced tolerance and response to antineoplastic treatments. Aside from this, cachexia directly accounts for nearly $20 \%$ of all cancer-related deaths, as the cachectic skeletal muscle wasting also affects survival needed muscles such as diaphragm and cardiac muscle, leading to respiratory and cardiac failure. In the last decade, the pivotal role of cancer-driven inflammation both in tumour progression and cachexia onset has clearly emerged, as well as cancer cachexia is associated to a broad range of metabolic and endocrine impairments leading to tissue function disruptions. Nevertheless, the potential role of muscle metabolic alterations in the activation and development of cancer cachexia has been little studied so far. Here, we report that conditioned media (CM) from murine and human carcinoma cell lines able to trigger cachexia in vivo, induce cachexia in $\mathrm{C} 2 \mathrm{C} 12$ myotubes that is associated to a metabolic shift towards fermentation. Our findings suggest that this metabolic shift is crucially involved in the activation of cachexia in C2C12 myotubes, since the abolishment through glycolysis block or lactate dehydrogenase inhibition, prevents the onset of the cachectic phenotype. Furthermore, our evidences suggest that $\mathrm{CM}$ from cancer cell lines able to induce cachexia express high levels of IFN-y and IL-6 and that these two proinflammatory cytokines could be involved in the metabolic shift towards fermentation that occurs in CM-treated myotubes, thus leading to cachectic phenotype. Overall, our results show the possible key role of the metabolic reprogramming towards fermentation in the induction of cachexia in myotubes, thus opening the study to new drugs that could counteract the metabolic shift and inhibit the cachectic phenotype. 


\begin{abstract}
6.5 PIN1: a putative molecular target to protect skeletal muscle against age-related muscle loss Lorenza Brocca ${ }^{a}$, Martina Grossob, Camilla Pezzinia, Valentina Semeghinib, Fiorenza Baruffaldib, Diletta Dolfinic, Adele Muccid, Valeria Righid, Pier Lorenzo Purie, Renata Battini ${ }^{b}$, Roberto Bottinellia, Maria Antonietta Pellegrino ${ }^{a}$, Susanna Molinarib

a. Dept. of Molecular Medicine, University of Pavia - b. Dept. of Life Sciences, University of Modena and Reggio Emilia, Modena- c. Dept. of Biosciences, University of Milano - d. Dept. of Chemical and Geological Sciences, University of Modena and Reggio Emilia, Modena - e. IRCCS Fondazione Santa Lucia, Rome, Italy.

Aging is associated with a progressive loss in skeletal muscle mass and strength, known as sarcopenia. Sarcopenia results in a decrease in mobility and an increased risk of developing chronic metabolic disease, thus it represents a major socio-economical problem. Age-related muscle loss cannot be consistently prevented by physical therapy and a pharmacologic therapy does not exist, probably because the molecular basis of this condition is still largely unknown. Many factors such as mitochondrial dysfunction, oxidative stress, inflammation, changes in the innervation of muscle fibers probably play an important role in age-related muscle decline. PIN1 is a widely expressed Peptydyl Prolyl cis/trans isomerase, involved in post-phosphorylation control of the function of multiple target proteins. Many evidences indicate that PIN1 controls signaling pathways involved in skeletal muscle wasting. Our results indicate that skeletal muscle of Pin1 KO mice is protected against muscle loss and weakness during aging. At the molecular level, we found 1) an increased expression of Peroxisome proliferator-activated receptor gamma coactivator 1-alpha (PGC1 $\alpha$ ), a transcription factor that promotes mitochondrial biogenesis, in skeletal muscle of Pin1 KO mice. Coherently with their resistance to muscle loss, we also found 2) an increase in the expression of the protein synthetic signaling proteins p70 ribosomal S6 kinase (S6K) and of its phosphorylated form in aged skeletal muscle of Pin1 KO mice compared to the wild type controls, suggesting that in these mice protein synthesis is maintained efficient. We also found 3) a significant decrease of Myostatin levels in skeletal muscle of aged Pin1 KO mice. It is well known that Myostatin is up regulated sarcopenia; it activates SMAD2/3 signaling and contributes to protein degradation and muscle atrophy. The transcriptional effects of Pin1 depletion on PGC1a and S6K genes must be mediated by a transcription factor. A putative candidate to mediate these effects is represented by the transcription factor Myocyte Enhancer Factor 2C (MEF2C), a known PIN1 target. In skeletal muscle cells a specific splice variant of MEF2C, MEF2C a1, activates the increase of skeletal muscle mass by activating the expression of IGF1 and S6K. These activities are repressed by its phosphorylation, that renders it a target for the inhibitory effect of PIN1 on its protein stability and activity. Coherently with these premises, we found 4) a decrease of MEF2C protein phosphorylation levels in aged KO mice compared to the control animals. This might at least partially contribute to the increased expression of PGC1 $\alpha$ and of S6K. Our results indicate that PIN1 could represent a valuable pharmacological target to counteract age-related muscle loss, simultaneously modulating multiple targets in a concerted way.
\end{abstract}

\title{
6.6 Involvement of CerK in skeletal muscle atrophy.
}

Federica Pieruccia ${ }^{a}$, Maria Chiara lachinia ${ }^{a}$ Alessia Fratia ${ }^{a}$ Chiara Battistinia ${ }^{a}$ Fabio Penna ${ }^{b}$, Paola Costelli $^{b}$, Elisabetta Meaccia

a. Dept. of Experimental and Clinical Biomedical Sciences "MarioSerio"-Unit of Biochemical Sciences and Molecular Biology, University of Florence - b. Dept. of Clinical and Biological Sciences, University of Turin, Italy

Skeletal muscle (SkM) atrophy is determined by several physiological and pathological conditions, such as cancer and neuromuscular disorders. Biomolecular characteristics of most types of SkM atrophy are the suppression of protein synthesis and the increase in protein degradation. Although the signalling pathways involved in the control of SkM waste are multiple, the regulatory role of lipids is only in part known. Sphingolipids (SLs) represent a class of bioactive molecules capable of modulating the destiny of many cell types, including SkM cells. Here, we investigated the role of ceramide kinase (CerK), the enzyme involved in the phosphorylation of Ceramide (Cer) to Ceramide 1-phosphate (C1P). We found that the expression of CerK was comparable in $\mathrm{C} 2 \mathrm{C} 12$ myoblasts and in terminally differentiated myotubes excluding that CerK expression modulators may be required for the achievement of myogenic differentiation. However, CerK inhibition promotes myoblast cell growth arrest and myotubes reduction in size suggesting a role of Cer/CerK axis in the maintenance of normal mature phenotype. Notably a drastic decrease of CerK protein expression was observed in SkM tissues obtained from animals bearing the C26 tumor, a well-characterized experimental model of cancer cachexia, and in $\mathrm{C} 2 \mathrm{C} 12$ myotubes treated with the glucocorticoid Dexamethasone. These findings provide the evidence that Cer/CerK axis acts as a molecular regulator of SkM atrophy, thereby representing a new possible target for therapy in pathophysiological muscle conditions. 


\subsection{Receptor for advanced glycation end-products (RAGE) as a biomarker of muscle wasting in cancer conditions

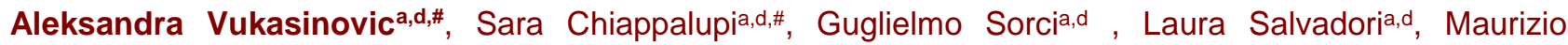

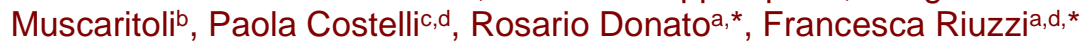

a. Dept of Experimental Medicine, University of Perugia - b. Dept of Clinical Medicine, Sapienza University of Rome - c. Department of Clinical and Biological Sciences, University of Turin - d. Interuniversity Institute of Myology (IIM), Italy - \# Equally contributed - * Shared senior authorship

Cachexia is a debilitating syndrome affecting the majority of patients with advanced cancer, and directly responsible for about $20 \%$ of all cancer-associated deaths. The major clinical feature of cachexia is skeletal muscle atrophy that leads to pronounced weight loss, drastically dampens patients' quality of life, reduces the response and tolerance to chemotherapy, and is associated with poor prognosis and outcome (1). The identification of reliable biomarkers of cachexia is of great importance to identify patients in the different phases of the disease and to monitor the treatment outcome (2). RAGE (receptor for advanced glycation end-products) is a multiligand receptor of the immunoglobulin superfamily physiologically involved in skeletal muscle development and homeostasis (3). In cancer conditions, the increase of RAGE ligands levels leads to hyperstimulation of the receptor translating into systemic inflammation and muscle wasting, and reducing mouse survival (4). We analysed RAGE expression in muscle tissue of different preclinical mouse models of cancer cachexia [Lewis lung carcinoma (LLC)-bearing C57BI/6 and colon adenocarcinoma (C26-ADK)-bearing $\mathrm{BALB} / \mathrm{c}$ mice] in the absence or presence of voluntary endurance exercise (wheel), which has been reported to partially antagonize muscle wasting in mice (5), and correlated RAGE expression with myofiber crosssectional area (CSA) and hallmarks of muscle atrophy (body and muscle weights, protein degradation extent, and activation of proteolytic systems). We also analysed RAGE expression in muscle biopsies from cancer patients. We found that: i) LLC- and C26-ADK-bearing mice express RAGE in myofibers in coincidence with reduced body and muscle weights and induction of proteolysis; ii) an inverse relationship exists between RAGE expression in muscles and tumor masses and the beneficial effects of endurance exercise in LLC-bearing mice; iii) RAGE expression increases in muscles during cachexia progression; iv) RAGE is not expressed in myofibers of athymic-nude mice injected subcutaneously with LLC or melanoma A375 cells, which do not develop cachexia; and $v$ ) muscles of cachectic patients express higher amounts of RAGE than non-cachectic subjects. Altogether, our results suggest that RAGE might represent a biomarker to monitor the cachectic stage at muscle level.

1) Porporato et al., Oncogenesis 2016, $5: \mathrm{e} 200$;

2) Loumaye and Thissen, Clin Biochem 2017, 50:1281-8

3) Riuzzi et al., J Cachexia Sarcopenia Muscle 2018, 9:1213-34;

4) Chiappalupi et al., J Cachexia Sarcopenia Muscle (under revision):

5) Aversa et al., Ther Adv Med Oncol 2017, 9:369-82. 


\subsection{A novel 3D culture system as an in vitro model for muscle disease}

Marianna Cosentino ${ }^{1}$, Carmine Nicoletti ${ }^{1}$, Simona Pisu², Emanuele Rizzuto ${ }^{2}$, Zaccaria Del Prete ${ }^{2}$, Antonio Musarò̀ 1.

1. DAHFMO-Unit of Histology and Medical Embryology - 2. Dept. of Mechanical and Aerospace Engineering, University of Rome "La Sapienza", Italy

Tissue engineering (TE) and regenerative medicine approaches are designed to increase skeletal muscle damage response. Scientific advances in biomaterials and stem cells allowed the opportunities to generate tissues from combinations of engineered extracellular matrices, cells and biologically active molecules in order to promote muscle regenerative response. Therefore, functional contractile properties as close as possible to that of healthy muscles are requested to allow for a good compatibility and a proper functional contribution. The X-MET is an engineering vascularized skeletal muscle tissue able to recapitulate the complex morphological properties, the architecture and the function of skeletal muscle. The X-MET shows biomechanical properties like muscles, can contract spontaneously as well as to respond to electrical stimulation. The aim of our study is to define the functional plasticity of X-MET subjected to mechanical stimuli. Interestingly, preliminary evidences suggest that different mechanical tensions induce a functional remodeling of X-MET toward a non-skeletal muscle phenotype. In this work, we demonstrated that X-MET show preserved cellular communication junctions, endogenous extracellular matrix (ECM), and integrative adhesive agents that could create a favorable environment for survival and integration of the X-MET in the host tissue. For this features, X-MET can be considered a useful experimental tool for in vitro and in vivo studies to improve drug discovery technologies, to supersede current preclinical animal models of disease and to restore the lost function of muscles.

\subsection{Use of Sertoli cells to treat DMD patients is supported by their immunomodulatory rather than immunosuppressive effect}

Sara Chiappalupia,c,\#, Laura Salvadoria,c,\#, Giovanni Luca ${ }^{a}$, Iva Aratoa, Francesca Mancusoa, Monica Borghia, Mario Calvittia, Francesca Riuzzia, ${ }^{\mathrm{a}, \mathrm{c}}$, Luigina Romania ${ }^{\mathrm{a}}$, Riccardo Calafiore ${ }^{\mathrm{b}}$, Rosario Donato ${ }^{\mathrm{a}, \mathrm{c}}$, Guglielmo Sorcia,c

a. Dept. Experimental Medicine - b. Dept. Medicine, University of Perugia - c. Interuniversity Institute of Myology (IIM) Italy - \# Equally contributed

Sertoli cells $(\mathrm{SeC})$ are the major component of the seminiferous tubules in the testes, where they secrete a plethora of trophic and immunomodulatory factors necessary for development of germ cells and to protect developing germ cells against the immune system attack, respectively. Naked or encapsulated $\mathrm{SeC}$ have been widely used as a therapeutic approach to many pre-clinical studies [1,2]. A single intraperitoneal (i.p.) injection of microencapsulated porcine $\mathrm{SeC}(\mathrm{SeC}-\mathrm{MC})$ translated into recovery of muscle morphology and performance in dystrophic $(m d x)$ mice in the absence of pharmacological immunosuppression, thanks to a double SeC effect, i.e., an antiinflammatory action and heregulin beta 1-dependent induction of utrophin at the sarcolemma [3]. This opened a new route of treatment for Duchenne muscular dystrophy (DMD) patients. However, it is still debating if $\mathrm{SeC}$ exert immunomodulatory or immunosuppressive effects, which is of relevance in view of their application in humans. Here we show that mice previously injected i.p. with SeC-MC were more able to solve pulmonary infection by Aspergillus fumigatus, one of the most common species causing disease in immunodeficient individuals, compared to control mice injected with empty microcapsules (E-MC), as evaluated by analysis of bronchoalveolar lavage, fungal growth, histology (PAS and Grocott staining), and inflammatory chemokines and cytokines expression (RT-PCR) in lungs. Moreover, mice previously injected i.p. with SeC-MC and later injected subcutaneously with LLC (Lewis lung carcinoma) cells showed similar tumor growth over time to mice injected with $\mathrm{E}-\mathrm{MC}$, further suggesting that $\mathrm{SeC}$ do not exert an immunosuppressive role. This was also confirmed by the observation that one year from i.p. injection SeCMC-injected $m d x$ mice did not show neither adverse effects nor increased incidence of tumor formation. Altogether, our data suggest that $\mathrm{SeC}$ exert immunomodulatory rather than immunosuppressive effect, further supporting the use of i.p. injection of SeC-MC as a potential treatment of DMD patients and diseases characterized by an inflammatory or autoimmunity environment.

[1] Luca et al. (2018) Sertoli cells for cell transplantation: preclinical studies and future perspectives. Andrology 6:385-95

[2] Chiappalupi et al. (2017) Employment of microencapsulated Sertoli cells as a new tool to treat Duchenne muscular dystrophy. J. Funct. Morphol. Kinesiol. 2(4):47

[3] Chiappalupi et al. (2016) Intraperitoneal injection of microencapsulated Sertoli cells restores muscle morphology and performance in dystrophic mice. Biomaterials 75:313-26 


\subsection{The voice of patients affected by Duchenne and Becker Muscular Dystrophies \\ Gloria Antonini}

Parent Project aps is an association of patients and parents of children affected by Duchenne and Becker Muscular Dystrophies (DMD and BMD), considered the most common among rare diseases and for which there is still no cure. The association is committed to funding research and disseminating the multidisciplinary approach that has so far enabled doubling the patients' life expectancy and improving their quality of life. Research support is the key objective of the Parent Project Association. The Scientific Office manages all activities related to the support of research and the dissemination of scientific information to patients, families and the outside world. The office also manages the Italian DMD/BMD patient registry, an integral part of the Global Registry established by the Treat-NMD, a network of excellence whose objective is the coordination and harmonization of research in the field of neuromuscular diseases. Like every year, Parent Project will organize, on the $28^{\text {th }}$ February $-1^{\text {st }}$ March 2020 in Rome, the International Conference on Duchenne and Becker Muscular Dystrophy where medical-scientific updates and dedicated spaces to families will be combined in a balanced way.

\subsection{Pterostilbene induces autophagic flux in Collagen VI-deficient muscle}

Samuele Metti $\mathrm{a}^{*}$, Lisa Gambarotto ${ }^{*}$, Martina Chrisamª, Martina La Spina ${ }^{b}$, Mauro Zorattib, Paolo Bonaldo ${ }^{a}$ a. Dept. of Molecular Medicine - b. Dept. of Biomedical Science, University of Padova, Italy - ${ }^{*}$ Equally contributing authors Autophagy is an evolutionarily-conserved self-eating mechanism, by which damaged or unnecessary intracellular components are subjected to lysosome-mediated degradation and recycling. Impaired autophagy was linked to several pathological conditions, including cancer, neurodegeneration and congenital muscle disorders. It is for this reason that autophagy upregulation, elicited, among others, by nutritional approaches, gained an increasing interest as therapeutic strategy. In in vitro study on murine primary dermal fibroblast, we found that Pterostilbene (Pt), a natural polyphenol chemically similar to Resveratrol but with higher bioavailable, is able to induce autophagic flux. Based on these data, we moved forward to an in vivo model of autophagy impairment, by performing a 5-day-long Pt treatment in Collagen VI null (Col6a1---) mice, a well-characterized model for COL6-related myopathies. Pt treatment strongly reactivates the autophagic flux in skeletal muscle, with a parallel decrease of the myofibers cross-sectional area and of apoptotic myonuclei. In agreement with this catabolic effect, lysosomal content and the mitophagic marker BNIP3 are increased. These findings demonstrate for the first time the capability of a member of the stilbenoid family to modulate autophagy in skeletal muscle. Further studies will allow to understand in detail the beneficial effects of Pt in ameliorating the myopathic defects and counteracting the muscle pathology of Collagen VI null mice.

\subsection{Inhibition of Acid Sphingomyelinase as Novel Alternative Therapy for Duchenne Muscular Dystrophy \\ Paulina Melania Roux-Biejat, Marco Coazzoli, Clara De Palma, Matteo Giovarelli, Emilio Clementi, Cristiana} Perrotta

Dept. of Biomedical and Clinical Sciences "Luigi Sacco" Università degli Studi di Milano

Duchenne Muscular Dystrophy (DMD), caused by genetic mutations leading to the absence of dystrophin, is the most common and severe muscular dystrophy, characterised by progressive muscle degeneration leading to premature death by the age of 20-30 years old. In DMD skeletal muscle, the repeated episodes of muscle fiber injury and necrosis, and the subsequent fibrosis are driven to a large extent by complex interactions between dystrophin deficiency and the host immune response. This has been demonstrated by removing macrophages and other inflammatory cell types in the $m d x$ mouse model of DMD as well as through the inhibition of key mediators of inflammation. The approved therapies, based on corticosteroids, support the evidence of the major role of inflammation in this disease. Nevertheless, due to their limitations in terms of efficacy and adverse events, they encourage us to find alternative approaches derived from the identification of novel suitable therapeutic targets. To this end we have been investigating, in DMD progression, the pathway of the sphingolipid metabolising enzyme, acid sphingomyelinase (A-SMase), which has recently been demonstrated to be involved in inflammatory related disorders. Our results in $m d x$ mice muscles revealed an upregulation of A-SMase expression and activity strongly connected to inflammation. Muscle regeneration in DMD can be impaired due to an imbalance in M1 (pro-inflammatory) and M2 (anti-inflammatory) macrophage phenotypes. Our studies in A-SMase $/$ mice, to investigate deeper the role of A-SMase show that this enzyme is fundamental for M1 macrophage polarization and that following injury in A-SMase $/$ mice there is a short M1 macrophage phenotype retention phase and a corresponding enhanced M2 macrophage phenotype function. As a proof-of-concept of pharmacological inhibition of A-SMase as a potential strategy for DMD we analysed 
A-SMase expression or activity in mice treated with drugs already proven to be beneficial for $m d x$ mice: the anti-inflammatory drugs naproxen/naproxcinod and amitriptyline, a functional inhibitor of A-SMase (FIASMA). We found that the anti-inflammatory drugs inhibited expression and activity of A-SMase while amitriptyline reduced the enzyme activity. Our next step is to test the in vivo efficacy of other FIASMAs (i.e. the SSRI fluoxetine and sertraline) with a better safety profile than amitriptyline. The advantage of this strategy is manifold: i. these drugs are already approved by the EMA and the FDA, confirmed minimally toxic and potentially rapidly available for clinical use as repositioned drugs; ii. they can also alleviate the depressive symptoms often displayed by patients; iii. they can synergise with the gene-based therapies currently under investigation.

\subsection{A novel wet-spinning system for 3D bioprinting of an artificial skeletal muscle tissue.}

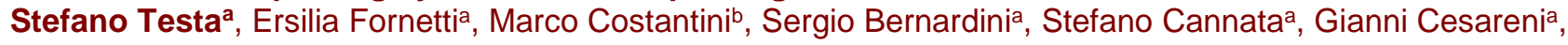
Cesare Gargiolia.

a. Dept. of Biology, University of Rome Tor Vergata, Italy - b. Faculty of Materials Science and Engineering, Warsaw University of Technology, Poland.

The skeletal muscle tissue exhibits regenerative capabilities related to damage extension. In fact, it is able to restore limited injuries while, following volumetric muscle loss (VML), the recovery is poor accompanied by scar formation and functional detriment. This condition negatively affects the quality of life of patients affected by VML, making necessary reconstructive therapeutic approaches. Even if surgical autologous transplantation is a standardized procedure, the outcomes are often unsatisfactory. Hence, the pressing need to develop engineered artificial tissues to replace wasted muscle. Tissue engineering (TE), exploiting stem cells embedded in biomimetic scaffolds, aims to mimic organogenesis by building artificial tissues to replace the damaged ones. Skeletal muscle TE is an up-and-coming biotechnology with great potential for muscle repair, but no conclusive strategy has been conceived yet. Reconstructing the skeletal muscle architecture and function is still a challenge requiring the parallel alignment of myofibrils arranged into organized sarcomeres. Recently we demonstrated the great potential of a hybrid biomimetic matrix, namely PEG-Fibrinogen, for enhancing the engraftment of myogenic cell progenitors by providing a suitable 3D environment for mouse muscle reconstruction. Starting from these observations, we chose to use a novel approach for the regeneration and/or reconstruction of skeletal muscle tissue combining muscle progenitors with 3D bio-printing technology to guarantee a functional architecture. In particular we have developed a microfluidic wet-spinning system that allows to fabricate rapidly macroscopic yarns of cell-laden hydrogel microfibers that closely mimic the structure of skeletal muscle tissue. In vitro and in vivo characterization of cell-laden constructs showed enhanced myogenesis and positive myo-structure alignment.

\subsection{Hitting DMD With a One-Two Punch: a Novel MAOB/VAP1 Inhibitor Greatly Improves the Pathological Phenotype in mdx Mice}

Lucia Tibaudo ${ }^{a, b}$, Leonardo Nogara ${ }^{a, c, d}$, Elena Germinarioa, Michela Bondia, Alberto Buson ${ }^{\mathrm{e}}$, Wolfgang Jarolimek $^{\mathrm{e}}$, Bert Blaauw ${ }^{\mathrm{a}, \mathrm{c}, \mathrm{d}}$, Libero Vitiello ${ }^{\mathrm{b}, \mathrm{c}}$, Marcella Canton , $^{\mathrm{a}} \mathrm{f}$

a. Dept. of Biomedical Sciences - b. Dept. of Biology, University of Padova - c. Interuniversity Institute of Myology (IIM) - d. Venetian Institute of Molecular Medicine (VIMM), Italy - e. Drug Discovery Dept., Pharmaxis Ltd., Sydney, NSW, Australia - f. Fondazione Istituto di Ricerca Pediatrica Città della Speranza - IRP, Padova, Italy

Oxidative stress and mitochondrial dysfunction play a crucial role in the pathophysiology of muscular dystrophies. We recently reported that the reactive oxygen species (ROS) produced by the mitochondrial enzyme monoamine oxidase B (MAO-B) are causally involved in Duchenne pathophysiology, as demonstrated by the results we obtained by treating mdx mice with the MAO-B specific inhibitor safinamide. We now show that a novel drug, PXS, designed to inhibit both MAO-B and the amino oxidase VAP-1 (a glycoprotein found on the plasma membrane of several cell types), provides comparable or superior effect to safinamide, but at a much lower dosage. Specifically, we found that a 30-day treatment with PXS in one- and three-month-old mdx mice greatly reduced fibrosis, inflammation and oxidative stress in both diaphragm and tibialis anterior, while also improving muscle contractile properties in diaphragm and gastrocnemius. Importantly, we have also obtained preliminary indications that a 90-day treatment with PXS led to a decrease in heart fibrosis in ninemonth-old animals. Considering that MAO-B inhibitors have been in clinical use for many years and that PXS has already passed all toxicity tests in several animal models, our findings indicate that PXS could be a promising candidate for the treatment of DMD. 


\section{POSTER \\ ABSTRACTS}


16th IIM Meeting (2019) - Forword \& Abstracts

Eur J Transl Myol 30 (3): 1-Yx, 2020 - doi:

P. 01. Lurbinectedin delays the onset of splenomegaly and extends survival of C26 tumor-bearing mice Giorgio Aquilaa ${ }^{a}$, Andrea David Re Cecconia, Giulia Terribilea, Roberta Frapollib, Ezia Bellob ${ }^{b}$, Deborah Novellic, Lidia Staszewskyc, Roberto Latinic, Maurizio D'Incalcib , Rosanna Piccirilloa

a. Dept. of Neuroscience - b. Dept. of Oncology - c. Dept. of Cardiovascular Medicine, Istituto di Ricerche Farmacologiche Mario Negri IRCCS, Milan, Italy

Lurbinectedin (PM01183, PM) is a synthetic alkaloid derivate of trabectedin (ET743, ET), a marine-derived anticancer agent. PM is a DNA minor groove covalent binder that has been tested in different Phase I-III trials. It affects tumor microenvironment by limiting the production of inflammatory cytokines. Some of these cytokines are elevated in various cancers characterized by rapid body wasting with muscle, fat and cardiac tissue depletion (i.e. cachexia). Differing from ET, PM displays less liver toxicity, and less endothelial inflammation at the site of injection. Mice injected with murine colon adenocarcinoma C26 cells display cachexia, increased circulating levels of inflammatory cytokines, acute phase response activation and subsequent splenomegaly. Thus, we tested whether PM, at doses with no antitumoral activity on C26 colon adenocarcinoma, has any beneficial effects in mice against C26-induced cachexia. 10-weeks old BALB/c mice were injected subcutaneously with $\mathrm{C} 26$ cells and three days later randomized to receive into their tail vein, three times a week for three weeks $0.07 \mathrm{mg} / \mathrm{kg} \mathrm{PM}(n=8)$ or vehicle $(n=8)$. C26-carrying mice showed decreased body weights and premature death. Strikingly, PM was able to extend the lifespan of C26-bearing mice by about $85 \%$ from a median survival time of 20 days (range day 10-31) to a median survival time of 37 (range day 14-41). This occurred without affecting tumor growth or food intake or the number of lung metastases. Another set of mice were sacrificed at 10-13 days from C26 implant. PM did not grossly protect multiple tissues (fat, muscle, heart) from cachexia. Preliminary data showed that C26-induced splenomegaly was inhibited by PM administration as long as PM treatment lasted. In C26-bearing mice, this effect exerted by PM seems to be associated also to restrained circulating levels of M-CSF, but not of other inflammatory cytokines (i.e. IL-6, G-CSF or GM-CSF). Further studied are necessary to correlate the improved survival with the pharmacodynamic effect observed.

\section{P. 02. Oxytocin as a physiological anti-cachectic agent?}

Alexandra Benoni ${ }^{1,2}$; Medhi Hassani ${ }^{1,2}$; Viviana Moresi ${ }^{1}$; Li Zhenlin ${ }^{2}$; Dario Coletti ${ }^{1,2}$; Xue Zhigang ${ }^{2}$; Sergio Adamo $^{1}$

1. DAHFMO Unit of Histology and Medical Embryology, and Interuniversity Institute of Myology, Sapienza University of Rome, Italy - 2. Dept. Of Biological Adaptation and Ageing B2A (CNRS UMR 8256 - INSERM ERL U1164 - UPMC P6), Sorbonne University, France Oxytocin, classically known for its effects on uterus, lactation, CNS, is also a powerful regulator of myogenic differentiation and muscular homeostasis. Previous works have shown that the addition of OT stimulates differentiation of myogenic precursors and induces myotube hypertrophy. Interestingly, in sarcopenia (senile muscular atrophy) exogenous OT antagonizes skeletal muscle atrophy and restores skeletal muscle trophism in mice. Neoplastic cachexia has a strong negative prognostic significance in cancer patients, being associated with a reduction in quality of life and response to therapies. Several pharmacological and hormonal treatment have been proposed to counteract cachexia, but this syndrome is still incurable. Oxytocin (OT) plays a physiological role in the maintenance of muscle homeostasis in aging. Therefore, we propose to study whether the administration of OT counteracts skeletal musculature atrophy in cancer-cachexia. To mimic cancer cellmediated effects on muscle cells, we incubated L6 myoblasts with C26 tumor-conditioned medium (C26 CM). Preliminary observations suggest that in vitro the inhibition of differentiation by $\mathrm{C} 26 \mathrm{CM}$ is reversed by the addition of OT in the culture medium of myogenic cells. We also showed that in vivo, OT counteracts the TNF effects on muscle regeneration of the Tibialis muscle following freeze-injury. Since hampered muscle regeneration and satellite cell function is a key feature of cachexia, contributing to muscle wasting, our preliminary data suggest that indeed OT treatment may have a beneficial effect on muscle homeostasis in tumor bearing mice. Thanks to the fact that OT is already approved for clinical use, this work seems promising to prevent cancer-cachexia skeletal muscle atrophy in patients.

\section{P. 03. Role of STAT3-mediated autophagy in driving muscle regeneration during aging} Giorgia Catarinella ${ }^{1,2}$, Andrea Bracaglia ${ }^{1}$, Elisa Bisicchia ${ }^{1}$, Francesca Di Felice ${ }^{1}$, Alessandra Sacco ${ }^{3}$, Lucia Latella ${ }^{1,2}$

1. Epigenetics and Regenerative Medicine, IRCCS Fondazione Santa Lucia, Rome - 2. Institute of Translational Pharmacology, National Research Council of Italy, Rome, Italy - 3. Development, Aging and Regeneration Program, Sanford Burnham Prebys Medical Discovery Institute, La Jolla, CA, USA

Age-related neuromuscular diseases are associated with a decline in muscle stem cell (MSC) function and an age-dependent muscle wasting, also called sarcopenia. Despite the clinical and social impact of sarcopenia, the age-related decline of muscle mass and function in not entirely deciphered. It has been demonstrated a key role of STAT3 in regulating MSC expansion and differentiation, extending its use as therapeutic target to 
16th IIM Meeting (2019) - Forword \& Abstracts

Eur J Transl Myol 30 (3): 1-Yx, 2020 - doi: ....

ameliorate muscle wasting. In addition, we recently described the essential role of autophagy in driving MSC function toward efficient muscle regeneration. The established role of autophagy in maintaining muscle mass and tissue homeostasis together with the emerging role of STAT3 in regulating the autophagic process inspired the rationale behind this work which resides in the study of the autophagic contribution in mediating STAT3 signalling toward skeletal muscle repair, a function that is compromised during aging. Our hypothesis is that STAT3 might have a role in regulating myogenic lineage and regeneration process by affecting the autophagic process thereby restoring the pro-myogenic niche that support muscle regeneration. We show that the autophagic process influences MSC activation and proliferation suggesting that autophagy might exert different functions depending on MSC proliferative vs. myogenic state and in muscle fibers. Likely, a combination of autophagy impairment in both compartments cooperates in muscle wasting during sarcopenia. Our further analysis indicates that STAT3 displays nuclear localization in conditions of active autophagy -i.e. young mice- while STAT3 localize in the cytoplasm in aged muscles, characterized by reduced autophagic process. Altogether, these evidences suggest that the nuclear/cytoplasmic compartmentalization of STAT3 regulates the autophagic process and the regenerative drive, highlighting potential biological targets that preclude an efficient regenerative response in aged mice.

\section{P. 04. Novel approaches to counteract Aging by physical intervention}

Ester Sara Di Filippo ${ }^{a, b}$, Danilo Bondia,b, Mariangela Marrone $e^{a, b}$, Tiziana Pietrangelo ${ }^{a, b}$, Stefania Fullea,b

a. Dept. of Neuroscience Imaging and Clinical Sciences, Laboratory of Functional Evaluation and Cellular Physiology, G. d'Annunzio University of Chieti-Pescara, Chieti, Italy - b. Interuniversity Institute of Myology, Italy

Ageing, in humans, is characterized by a progressive loss of muscle mass and strength, known as sarcopenia. In the sarcopenic muscle, the regenerative capacity of satellite cells (SCs), adult muscle stem cells, is compromised. The SCs are responsible for the postnatal muscle growth and the maintenance of muscular mass in the adulthood. These cells are the main source of muscle stem cells able to regenerate the skeletal muscle tissue. During aging, the myogenic capability of SCs decreased and it results in the failure to complete the differentiation program. However, the mechanisms behind the impaired myogenic differentiation process of elderly are not fully understood. Moreover, during ageing in the skeletal muscle, there is an imbalance between reactive oxygen species (ROS) production and antioxidant enzyme activity and alterations in the gene expression ${ }^{1}$. The oxidative stress is harmful for the maintenance of skeletal muscle structure and function, and is considered one of the main contributors causing cellular aging. An effective exercise protocol might be particularly useful to counteract the detrimental decline that occurs in sarcopenic muscle. Indeed, the exercise could promote cellular and molecular adaptation mediated by microRNAs modulation ${ }^{2}$. Furthermore, we showed that during aging activated SCs display a complex picture of impaired regenerative potential, linked to altered ROS production and miR-1 and miR-133 dysregulation ${ }^{3}$. The aim of this study was to determine whether endurance and resistance training and neuromuscular electrical stimulation (NMES) affect muscle regeneration modulating microRNAs and oxidative management in healthy elderly. To achieve this goal, healthy male elderly subjects $(76.6 \pm 3.7$ years; $n=50)$ volunteers were recruited and randomly assigned to different training and control groups; training plans consisted in 12 weeks, 3 sessions/w. Functional evaluation tests revealed specific improvements of elderly performance. Furthermore, at the volunteer were done the skeletal muscle biopsy before (Pre-) and after (Post-) training. In detailed 1) Pre- and Post-SCs were isolated and characterized; 2) myo-microRNAs (miR133a, miR133b, miR-1, miR206) were analyzed; 3) oxidative status were assessed. In conclusion, our data suggested that endurance and NMES protocols promoted a higher antioxidant status. Strikingly, we found that physical intervention is able to modify myo-microRNAs expression. Indeed, they were specifically down- or up-regulated by the training typologies.

1. Fulle S et al. Exp Gerontol. 2005 Mar;40(3):189-97.

2. Di Filippo ES et al. J Appl Physiol (1985). 2017 Sep 1;123(3):501-512.

3. Di Filippo ES et al. Biochem Biophys Res Commun. 2016 Apr 29;473(2):462-70.

P. 05. Inhibition of $\mathrm{N}$-glycosylation impairs myoblast differentiation and IGF-1 receptor signalling pathways activation

Giosuè Annibalinia ${ }^{a}$ Laura Di Patriaa ${ }^{a}$, Roberta Saltarellia, Amelia Morrone ${ }^{b}$, Lorenzo Ferrib, Renzo Guerrinib, Serena Maggio ${ }^{a}$, Michele Guescinia, Vilberto Stocchia, Elena Barbieria,c

a. Dept. of Biomolecular Sciences, University of Urbino Carlo Bo, 61029 Urbino, Ital; - b. Molecular and Cell Biology Laboratory, Dept. of Neurosciences, Psychology, Pharmacology and Child Health University of Florence and Pediatric Neurology Unit, Meyer Children's Hospital Florence 50139, Italy - c. IIM, Interuniversity Institute of Myology, Italy

Congenital disorders of glycosylation (CDG) are rare genetic disorders caused by abnormalities in the glycan biosynthesis [1]. CDG are associated with a broad range of clinical symptoms. Hypotonia is present at least in some CDG types, often in association with proximal muscle weakness [2]. Moreover, muscle biopsy of patients with PMM2-CDG, the most common form of CDG, showed mild myopathic alterations with variation in fiber size and myofibrillar disarray [3]. Here, we used $\mathrm{C} 2 \mathrm{C} 12$ cell culture model to investigate the effects of $\mathrm{N}$ - 
16th IIM Meeting (2019) - Forword \& Abstracts

Eur J Transl Myol 30 (3): 1-Yx, 2020 - doi: ....

glycosylation inhibition on myoblast differentiation. $\mathrm{C} 2 \mathrm{C} 12$ cells were treated with the $\mathrm{N}$-glycosylation inhibitor tunicamycin (TM) $(0.01 \mu \mathrm{g} / \mathrm{ml})$ and the mRNA expression of CCND1, Pax7, Myogenin, MyoD, MRF4 and Desmin was quantified in proliferative myoblasts and differentiated myotubes. PCNA, myosin (MF20), IGF-1R, IGF-1R pathway activation and myogenic index were also quantified by western blot and fluorescent imaging, respectively. Finally, we tested whether the siRNA knockdown of the PMM2 gene produces effects on myoblast differentiation resembling the TM administration. As expected, CCND1 and Pax7 were expressed in control myoblasts and were downregulated in myotubes, while Myogenin and Desmin showed the opposite trend. TMtreated myoblasts failed to down-regulate CCND1 mRNA after induction of differentiation and the expression of CCND1 and Pax7 remained higher in TM-treated compared to control myotubes. Conversely, TM-treated myotubes expressed lower level of Myogenin and Desmin compared to control. Moreover, MF20 protein and myogenic index were significantly reduced in TM-treated myotubes. Glycosylation inhibition also decreased the IGF-1R level and markedly attenuated the IGF-1-induced ERK-1/2 and Akt phosphorylation. Interestingly, $70 \%$ mRNA knockdown of PMM2 increased CCND1 in myoblasts and reduced Myogenin, MyoD and MRF4 mRNAs and MF20 expression in myotubes. In conclusion, we found a reduction of myoblast differentiation after N-glycosylation inhibition by TM and PMM2 silencing. TM administration also affected the IGF-1R pathway reducing the IGF-1R expression and inhibiting ERK-1/2 and Akt activation. The decrement in the expression of differentiation markers observed here after PMM2 silencing, suggests that a deficit in muscle cell differentiation could be present in CDG patients.

[1] Jaeken J. et al., J Inherit Metab Dis. 2017 40, 569

[2] Harris S.R. Developmental Medicine \& Child Neurology. 2008 50, 889

[3] Aronica E. et al. Acta Neuropathol. 2005 109, 433

\section{P. 06. Differential expression and epigenetic modulation of TGF- $\beta$ target genes in healthy and DMD} skeletal muscle cells

Monica Forino, Valeria Spadotto, Christian Steinkuhler, Gianluca Fossati

Italfarmaco SpA, Preclinical R\&D Dept., Italy

Duchenne Muscular Dystrophy (DMD) is an X-linked genetic disorder resulting from mutations and deletions of the dystrophin gene that leads to severe degeneration of muscle tissue that is progressively substituted by fat and fibrous tissue. Among the cytokines involved in this process, TGF- $\beta$ is a key player polypeptide that orchestrates the crosstalk between parenchymal, inflammatory and collagen-expressing cells with a recognized pro-fibrotic role. In DMD patients, TGF- $\beta$ is elevated both in plasma and in muscles and its expression correlates with increased fibrosis. In vitro, it has been found highly expressed in DMD myotubes and in muscle derived fibroblasts. TGF- $\beta$ activity is strictly controlled at various levels and the cytokine is secreted as a latent complex where it is tightly bound to the so-called latent TGF- $\beta$ binding protein (LTBP) and released as active polypeptide upon specific proteolytic cleavage. Among the LTBPs described, the LTBP4 gene was discovered as a modifier of murine muscular dystrophy. Studies in DMD patients revealed that four LTBP4 SNPs were predictive of the age at onset of loss of ambulation and of dilated cardiomyopathy. Further studies in mice and cell culture demonstrated that more TGF- $\beta$ is released from the LTBP4 VTTT variant compared to the IAAM-containing LTBP4 variant, the latter being associated with older age at loss of ambulation and less TGF- $\beta$ signaling. Histone deacetylases (HDACs) are involved in fibrogenesis and reduction of HDAC activity by inhibitors targeting both class I and II, hinders TGF- $\beta$ induced fibrosis. Moreover, it was observed that our proprietary HDAC inhibitor givinostat significantly reduced fibrosis in $\mathrm{mdx}$ mice and in DMD boys. The aim of this study was to investigate the molecular effects of givinostat on TGF- $\beta$ induced fibrotic gene program in an in vitro model of human healthy and DMD-derived skeletal muscle cells. In agreement with its role, TGF- $\beta$ induced the expression of pro-fibrotic genes such as alpha smooth muscle actin ( $\alpha \mathrm{SMA})$, fibronectin (FN1) and collagen III, both in healthy and DMD cells. Givinostat clearly reduced the upregulation of $\alpha \mathrm{SMA}$ and FN1 in healthy cells while the effect on DMD cells was negligible for $\alpha \mathrm{SMA}$, and FN1 expression was further increased. Downregulation of collagen III was observed in both cell types. A different epigenetic regulation of key genes in the TGF- $\beta$ pathway was also highlighted by the effects on the expression of TGF- $\beta$ receptor subunits TGF R1 an R2. Thus, TGF R1 was upregulated by the cytokine and downregulated by givinostat in both cell types while TGF R2 was downmodulated by TGF- $\beta$ in DMD cells only and givinostat increased its expression in both cell types. A differential regulation of matrix metalloproteinases and their inhibitors was also observed. These data emphasize how a different epigenetic regulation of key fibrotic genes takes place in healthy and DMD cells. Furthermore, they also highlight the importance of having an in vitro model whereby both healthy and DMD cells are used to study the specific pathways that can be targeted in diseased cells to more accurately advance drug candidates into in vivo studies.

\section{P. 07. miRNAs as potential molecular biomarkers of cancer cachexia}


16th IIM Meeting (2019) - Forword \& Abstracts

Eur J Transl Myol 30 (3): 1-Yx, 2020 - doi:

Lorena Garcia-Castillo, Marc Beltrà, Fabrizio Pin, Giovanni Birolo, Barbara Pardini, Giuseppe Matullo, Fabio Penna, Paola Costelli

Dept. of Clinical and Biological Sciences, University of Turin, Italy - Interuniversity Institute of Myology, Italy

Introduction: Cancer-associated cachexia is a multifactorial syndrome characterized by anorexia and body weight loss, mainly due to muscle and fat wasting (1). MicroRNAs (miRNAs) are a class of non-coding posttranscriptional regulators that play, among other functions, a central role in appropriated muscle cell commitment, proliferation and differentiation by modulating the translation of specific genes (2). The present study was aimed to evaluate the possibility to use dysregulated miRNAs as biomarkers of cancer cachexia. Methods: Total RNA was extracted from both skeletal muscles and plasma-derived microvesicles from controls and C26 tumor-bearing Balb/c mice. Next-generation sequencing (NGS) was used to sequence whole miRNA transcriptome. Those miRNAs differentially regulated were validated by Real Time PCR. Results: Clear differences in miRNA expression between the two experimental groups were observed. In particular, miR-21a$5 p$, miR-29a-3p, miR-29c-3p, miR-185-5p, miR-185-3p, miR-223-3p were statistically upregulated in the tumor-bearing mice. By contrast, miRNAs from microvesicles were poorly modulated in the C26 host, only three miRNAs (miR-181a-5p, miR-375-3p, miR-455-5p) were found dysregulated at NGS analysis, suggesting that their use as a circulating biomarker might be limited.

Conclusion: The results obtained in the present study suggest that modulations of the skeletal muscle miRNAome can be used as effective biomarkers in the pathogenesis of cancer-associated cachexia in murine models.

1 V.E. Baracos, L. Martin, M. Korc, D.C. Guttridge, K.C.H. Fearon, Cancer-associated cachexia, Nat. Rev. Dis. Prim. 4 (2018), 17105. https://doi.org/10.1038/nrdp.2017.105

2 Tyler J. Kirby, Thomas Chaillou, John J. McCarthy. The role of microRNAs in skeletal muscle health and disease. Front Biosci (2016); 20: $37-77$

\section{P. 08. SCA1 expression variability governs Fibro/adipogenic progenitor (FAP) behaviour}

Giulio Giuliania, Claudia Fuocoa, Simone Vumbacaa, Ezio Giordab, Mara Vincib, Luisa Castagnolia, Gianni Cesarenia

a. Dept. of Biology, University of Rome Tor Vergata, Italy - b. Bambino Gesù Children's Hospital-IRCCS, Italy

The emerging awareness that cell populations are heterogeneous has changed our criteria to define a cell type. Among the non-genetic sources of cell to cell variability, a spread in a bell-shaped distribution of a feature defines a condition called micro-heterogeneity. The effect at cellular and physiological level of this type of heterogeneity remains to be investigated. We chose skeletal muscle regeneration and fibro/adipogenic progenitors (FAPs) to address this issue. FAPs are a population of mesenchymal progenitors that resides in the interstitium of skeletal muscle and are identified by the expression of SCA1. In the mdx mouse, an animal model of Duchenne Muscular Dystrophy (DMD), FAPs are one of the populations responsible for the development of ectopic tissues, such as intramuscular adipose tissue (IMAT) and fibrotic tissue. Taking advantage of a multiplex flow cytometry analysis we defined two FAP cell states characterised by high and low SCA1 expression (SCA1 High and SCA1 Low). These two sub-populations are differentially represented in wild type and $\mathrm{mdx}$ mouse. Next, we isolated the two FAP sub-populations by fluorescence activated cell sorting (FACS) from mdx mouse and we studied their differentiation potential ex vivo. We found that SCA1 High FAPs have a higher capability to express PPAR-gamma and to differentiate into mature adipocytes. Whereas, SCA1 Low FAPs differentiate readily into myofibroblasts in a pro-fibrogenic environment. Moreover, SCA1 High FAPs exhibit a higher proliferation rate than SCA1 Low cells. Overall, we demonstrate that micro-heterogeneity contributes to determine the fate of a mesenchymal population in vitro. These findings raise several questions about its interaction with in vivo microenvironment and its impact in pathology progression.

P. 09. SRF is a mechano-transductor in response to exercise and may play a role in the exercisemediated rescue of muscle homeostasis in cancer cachexia

Hassani Medhia, b, Benoni Alexandra ${ }^{a}$, Xue Zhigang ${ }^{b}$, Sotiropoulos Athanassiac, Parlakian Ara ${ }^{\mathrm{b}}$, Li Zhenlin ${ }^{\mathrm{b}}$, Adamo Sergioa, Coletti Darioa, b

a. DAHFMO Unit of Histology and Medical Embryology, and Interuniversity Institute of Myology, Sapienza University of Rome, Italy - b. Dept. Of Biological Adaptation and Ageing B2A (CNRS UMR 8256 - INSERM ERL U1164 - UPMC P6), Sorbonne University Paris 6 , France - c. Institut National de la Santé et de la Recherche Médicale, U810, and Faculté de Médecine Necker-Enfants Malades, Université Paris 5, France

Cancer-cachexia results in severe muscle tissue wasting affecting patients' quality of life and survival. Recent studies showed that physical activity increased survival in cancer patient and animal models. The underlying mechanisms, however, are still largely unknown. To identify signaling pathways involved in exercisedependent maintenance of muscle mass and function in cachexia, we investigated the role of serum response factor (SRF), a transcription factor of the MADS-box family, having a major role in muscular growth, differentiation and regeneration. We hypothesize that the expression and/or transcriptional activity of SRF 
16th IIM Meeting (2019) - Forword \& Abstracts

Eur J Transl Myol 30 (3): 1-Yx, 2020 - doi: ....

decreases in C26 tumor bearing mice, and that exercise can rescue. Indeed, we showed a decrease of SRF expression at the protein level in cancer cachexia. Consistently, we showed a decrease in the expression of SRF target genes such as MyoD and SK-Actin, which suggest a decrease of the SRF transcriptional activity. These effects were counteracted by wheel running. Since we observed opposite effects of tumor and exercise on MyoD and Pax7 we hypothesized the involvement of a myogenic program in stem cell recruitment to muscle fibers upon exercise: indeed, we observed the recruitment of nuclei within the muscle fibers in response to exercise, which could contribute to muscle homeostasis. Our preliminary results suggest that physical activity rescues SRF expression as well as its transcriptional activity, highlighting the importance of genetic activation induced by skeletal muscle activity for muscle rescue and homeostasis. These effects could be extended to the fiber microenvironment, including myogenic stem cell activity.

\section{P. 10. Sphingolipids regulate VDR expression in skeletal muscle cells \\ Maria Chiara lachini, Federica Pierucci, Elisabetta Meacci \\ Dept. of Biomedical Experimental and Clinical Sciences 'Mario Serio', University of Florence, Italy}

Vitamin (Vit) $D$ is a pleiotropic hormone synthesized mainly in the skin via a UV-dependent reaction. VitD circulates in the blood bound to vitamin D-binding proteins, reaching its target tissues, where exerts its endocrine actions through the VitD3 receptor (VDR), a member of the nuclear receptor family expressed in different tissues, including skeletal muscle. Some VitD actions have been shown to be at long term and others at more immediate term, suggesting the engagement of nuclear VDR for gene expression regulation and of membrane-associated VDR for the control of specific signalling pathways. VitD deficiency has been shown to cause abnormalities in skeletal muscle, such as reduced actomyosin content and decrease in mitochondrial $\mathrm{Ca}^{2+}$ levels. Moreover, in vitro experiments suggest that VDR is likely to play an important role during myoblast differentiation, regulating the expression of myogenin. Despite several evidence demonstrating a protective role of VitD in skeletal muscle cells, the characterization of biomolecules that positively affect VDR expression /function are limited. In the present study we investigate the role of sphingolipids in the regulation of VDR expression in $\mathrm{C} 2 \mathrm{C} 12$ myoblasts/myotubes and the molecular mechanisms by which SLs metabolites, in particular Sphingosine-1-phosphate, determine an increase in VDR expression in skeletal muscle cells.

P. 11. High levels of circulating Interleukin-6 affect the redox balance in skeletal muscle, inducing the expression of inflammatory-related factors

Carmen Miano*, Laura Forcina*, Carmine Nicoletti, Laura Pelosi, Antonio Musarò ( ${ }^{*}$ Co - First Author) Institute Pasteur Cenci-Bolognetti (DAHFMO-Unit of Histology and Medical Embryology, IIM, Sapienza University of Rome, Rome, Italy) Oxidative stress and chronic inflammation have been proposed as important interconnected mechanisms affecting skeletal muscle homeostasis under pathologic conditions, being mutually induced processes. Although reactive species are physiologically formed inside of skeletal muscle by inflammatory cells as well as myofibers, the endogenous antioxidant defense is able to maintain reactive radicals at functional levels. Instead, under pathological conditions the excess of reactive species can overwhelm antioxidant defense leading to oxidative damages and to the activation of pro-inflammatory pathways. On the other hand, inflammatory response is considered one of the principal determinants of degenerative processes in degenerative disorders. However, the clear connection between the extent of oxidative stress and inflammation has still to be understood. Hence the needs of identifying and studying key factors involved in the intimate relation between these two important pathogenic mechanisms that might allow relevant advances in the field of chronic diseases. Among factors playing a critical role in skeletal muscle physiopathology Interleukin-6 (IL6 ) is an elective candidate. Here we reported that increased levels of circulating IL-6 are sufficient to alter the physiologic redox balance of skeletal muscle tissue and to induce the establishment of an inflammatory milieu. We revealed that high levels of IL-6 in the bloodstream enhance the production and accumulation of reactive species in diaphragm muscles of adult NSE/IL- 6 mice, by impinging the muscle redox balance and impairing the Nrf2-mediated antioxidant response. In addition, the occurrence of pro-oxidant conditions in muscles exposed to abnormal amounts of IL-6 stimulates the establishment of a delicate inflammatory balance within muscle environment, characterized by the induction of pro-inflammatory mediators. Thus, uncontrolled levels of this cytokine, which characterize chronic degenerative conditions, can alter muscle milieu, linking the generation of oxidative stimuli to the promotion of inflammatory changes.

P. 12. The chromatin remodeling protein WDR5 is required for the aberrant expression of DUX4 in Facioscapulohumeral Muscular Dystrophy 
16th IIM Meeting (2019) - Forword \& Abstracts

Eur J Transl Myol 30 (3): 1-Yx, 2020 - doi:

Emanuele Mocciaro, Roberto Giambruno, Stefano Micheloni, Cristina Consonni, Maria Pannese, Valeria Runfola, Giulia Ferri, Davide Gabellini

Gene Expression and Muscular Dystrophy Unit, Division of Genetics and Cell Biology, IRCCS San Raffaele Scientific Institute, Milano, Italy

Background: Facioscapulohumeral Muscular Dystrophy (FSHD) is a frequent neuromuscular disease caused by epigenetic alterations on the $4 q 35$ chromosome leading to gain of expression of the double homeobox 4 (DUX4) gene. Our laboratory previously showed that the long non-coding RNA DBE-T is required for aberrant DUX4 expression in FSHD. Using affinity purification followed by proteomics, we identified the chromatin remodeling protein WDR5, a core component of the MLL/SET1 epigenetic activation complex, as a novel DBET interactor. The goal of this work is to analyze the role of WDR5 on the regulation of DUX4 in FSHD. Methods: Primary myoblast isolated from both FSHD and healthy control subjects were differentiated in myotubes in presence of siRNAs. 24 hours after induction of differentiation, myotubes have been transfected with siRNA on-target WDR5 and siRNA non-targeting as control. Four days after differentiation in mature myotubes the expression level of DUX4 and its known targets have been assessed with qRT-PCR. Moreover, the myogenic differentiating level and the fusion index have been assessed through immunofluorescence analysis of the myosin heavy chain (MHC) differentiation marker. Results: We found that the expression level of DUX4 and its target MBD3L2, TRIM43 and TRIM48 are significantly reduced by silencing WDR5. Similar results were obtained using a WDR5 small molecule inhibitor. Remarkably, WDR5 knockdown leads to rescue of the myogenic defects of FSHD muscle cells at both the differentiation level and the fusion index. Conclusion: These results show the pivotal role played by WDR5 in DUX4 expression and candidate WDR5 as potential therapeutic target for FSHD treatment.

P. 13. Extremely low frequency electromagnetic fields modulate the strength in skeletal muscle in sedentary adult mice

Caterina Morabito, Simone Guarnieri, Maria A. Mariggiò

Dept of Neuroscience, Imaging and Clinical Sciences; Centro Scienze dell' Invecchiamento e Medicina Traslazionale (CeSI-MeT), Univ. "G. d'Annunzio" of Chieti-Pescara, Italy

Many studies have been performed on understanding the mechanisms that regulate the relationships between extremely low frequency electromagnetic fields (ELF-EMFs) and the living matter. Initially, the attention of scientists was focused almost exclusively on the possible harmful effects triggered by the ELF-EMFs exposure; more recently many scientific papers reported possible therapeutic effects of these stimuli, consequently the definition of their possible mechanisms of action is currently a fascinating issue. Our previous studies have provided new insight into the mechanism(s) responsible for the biological effects of ELF-EMFs revealing that they affect intracellular reactive oxygen species production and myogenesis progression in C2C12 myoblasts, an in vitro skeletal muscle model (Morabito et al, Free Rad Biol Med 48:579-589, 2010; Morabito et al, BioMed Res Int 2017:2460215, 2017). The purpose of this work has been to study the skeletal muscle strength development, the main feature of this tissue, when sedentary adult mice were exposed to ELF-EMFs. Male C57BL/6 sedentary adult mice were exposed to ELF-EMFs ( 0.1 or $1 \mathrm{mT})$ generated by a solenoid for $1 \mathrm{~h} / \mathrm{die}$ up to 5 weeks and fed with a standard diet or a N-acetyl-cysteine (NAC) enriched diet. Grip strength and body weight were measured weekly, and after 5 week exposure the mice were sacrificed and skeletal muscle from hind limbs were isolated and collected for biochemical analyses. The ELF-EMFs did not affect the body weight and, conversely, increased the grip strength in exposed mice in comparison to non-exposed ones. In addition, both the expression levels of Pax7 and of myosin heavy chain were found increased in muscle isolated from exposed mice. The ELF-EMFs failed to trigger their effects when the mice were fed with an NAC-enriched diet. These preliminary data suggest that ELF-EMFs may provide some benefits improving the muscle fitness in sedentary adult mice.

P. 14. Inflammation in dystrophic heart and diaphragm: a comprehensive study Jacopo Morroni, Anna Benedetti, Rosanna Di Maggio, Biliana Lozanoska-Ochser, Marina Bouchè SAIMLAL Dept., Sapienza University of Rome

The current leading cause of death in Duchenne's Muscular Dystrophy (DMD) patients is cardio-respiratory failure, mainly due to heart and diaphragm (DIA) damage and fibrosis during the late stage of the disease. Given that fibrosis is considered a consequence of muscle inflammation, the role of the immune response in DMD heart and diaphragm should be considered, to better understand the pathological events during and prior to the onset of fibrosis. Indeed, very little is known about the kinetics of immune cells infiltration in dystrophic heart and diaphragm, that likely contributes to the fibrosis and chronic inflammation. The treatment of choice in dystrophic patient is the use of corticosteroids, supporting an important role of the inflammatory compartment in the disease progress. The several and severe adverse effects of long-term corticosteroid treatment also highlight the need for other anti-inflammatory approaches. Our group recently found that pharmaceutical 
16th IIM Meeting (2019) - Forword \& Abstracts

Eur J Transl Myol 30 (3): 1-Yx, 2020 - doi:

inhibition of Protein Kinase $C$ theta prevented damage in limb skeletal muscle and ameliorated disease when administered in young (2week-old) $m d x$ mice, acting predominantly through inhibition of early $T$ cell infiltration of dystrophic muscle. We now plan to study in detail the inflammatory compartment of dystrophic heart and DIA using the mouse model of DMD, $m d x$. We will characterize in detail the kinetics and quality of inflammatory cell populations in $m d x$ heart and DIA, using our established 9 color cytofluorimetric protocol, analysing mice from 4 weeks of age up to 11 months. Morphological analysis on muscle fibrosis, necrosis and organization will be performed, as well as molecular analyses to assess cytokines levels and fibrosis markers. Given that $m d x$ mice often show a poor heart pathology, we are currently defining an exercise protocol in order to worsen the dystrophic phenotype, in order to mirror the human pathology. The data we will collect in this part of the study will be instrumental to design new pharmacological treatments using specific inhibitors (as we did for skeletal muscle) instead of broad-spectrum anti-inflammatory compounds. We hope that our study will help developing more effective and fine targeted treatments, with less adverse effects for patients

P. 15. Intracellular attenuation of thyroid hormone influences energy metabolism by reducing mitochondria biogenesis and inducing mitochondrial dysfunction

Annarita Nappi ${ }^{\mathrm{a}}$, Annunziata Gaetana Cicatielloa, Serena Sagliocchia, Caterina Miroa, Emery Di Ciccoa, Giuseppina Mancinoa, Domenico Salvatore ${ }^{b}$, Monica Denticea

a. Dept. of Clinical Medicine and Surgery - b. Dept. of Public Health, University of Naples Federico II, Italy

Thyroid hormone $(\mathrm{TH})$ has a major role in the control of systemic metabolism, influencing carbohydrate, protein and lipid metabolism. Moreover, T3 regulates mitochondrial function and turnover controlling mitochondrial biogenesis, proton leak, OXPHOS and ROS generation. The deiodinases enzymes enable intracellular TH activation or inactivation, regardless of circulating hormone levels. To address the physiological function of deiodinases in skeletal muscle metabolism, we used muscle-specific gain-of-function approaches. Here, we show that muscle-specific hypothyroidism in mice by D3 overexpression (TG-D3 mice) induces a metabolic reprograming of muscle fibers reducing glycolytic and lipid metabolism. D3 overexpression in muscle also reduces mitochondrial dynamics. Immunofluorescence analysis revealed a structural alteration of mitochondria. FACS analysis highlighted a reduced mitochondrial density with increased size. However, the increased mitochondrial size did not correlate with increased activity. Consistently with these findings, mitochondria turnover genes DRP-1, MFN-1 and OPA-1, as well as PGC-1 $\alpha$, were reduced in TG-D3 muscles. These data suggest that metabolic differences between TG-D3 and control muscles are associated with an alteration in number and function of the mitochondria. Interestingly, the overexpression of PGC-1a in TG-D3 muscles rescued the mitochondrial defects, reactivating the expression of genes involved in mitochondrial fusion and fission. Altogether these results indicate that the mitochondrial dysfunction of TG-D3 muscles is mediated by the downregulation of PGC-1a, the master regulator of mitochondrial biogenesis. Our findings indicate that muscle-specific hypothyroidism via D3 overexpression potently impacts on mitochondrial metabolism, identifying the deiodinases as critical metabolic regulators. Understanding the mechanisms of action of deiodinases in metabolism might be relevant for therapeutic treatment of metabolic disorders.

P. 16. Musclin, a myokine induced by aerobic exercise, retards muscle atrophy during cancer cachexia Andrea David Re Cecconi ${ }^{\mathrm{a}}$, Mara Fortia, Michela Chiappa ${ }^{\mathrm{a}}$, Luigi Cervo ${ }^{\mathrm{a}}$, Luca Beltrame ${ }^{\mathrm{b}}$, Sergio Marchinib Rosanna Piccirilloa

a. Dept. of Neurosciences - b. Dept. of Oncology, Mario Negri Institute for Pharmacological Research IRCCS, Milan, Italy.

Background: Physical activity ameliorates the prognosis of cancer patients, also by contrasting the associated muscle wasting (i.e. cachexia). Since aerobic exercise seems to be the most effective to preserve muscles during cancer, we asked whether it promotes secretion of proteins by muscles (i.e. myokines) that may contrast cachexia. Methods: To mimic aerobic exercise, we infected $\mathrm{C} 2 \mathrm{C} 12$ myotubes with PGC1a expressing adenoviruses. In vitro we evaluated the effects of supernatants from GFP or PGC1a-overexpressing cells on protein synthesis and degradation of atrophying myotubes and in LucAssay. By microarray, we identified putatively secreted proteins inducible by PGC1 $\alpha$ and confirmed by Q-PCR. We measured by Q-PCR their expression in Tibialis Anterior (TA) muscle of C26-bearing mice during cachexia and plasma levels by ELISA. To induce aerobic exercise adaptations, mice were run on treadmill. Anaerobic exercise-like effects were obtained in vivo in overloaded Plantaris muscle and in vitro in myotubes expressing myristoylated AKT. Results: Our microarray and Q-PCR analyses showed musclin as a PGC1 $\alpha$-induced myokine. Conversely, its expression was unchanged in myotubes hypertrophying because of activated AKT. Dexamethazone-treated myotubes or constitutively active (ca)FoxO3-expressing ones undergo atrophy as measured by increased proteolysis and MuRF1 induction. Unlike to GFP, musclin restrained the dexamethazone-induced MuRF1 expression in Luciferase assays. Consistently, musclin-containing supernatants reduced the caFoxO3-induced rates of long-lived protein degradation. Among the newly identified PGC1 $\alpha$-induced myokines, we found that 
16th IIM Meeting (2019) - Forword \& Abstracts

Eur J Transl Myol 30 (3): 1-Yx, 2020 - doi:

only musclin (and its receptor Npr3) was strongly downregulated in cachectic muscles and plasma of C26bearing mice. Thus, we electroporated TA of C26-bearing mice with musclin or Npr3-encoding plasmids and found either musclin or Npr3 to preserve fiber area. Interestingly, treadmill exercise protected C26-bearing mice from muscle loss, with no effect on tumor growth, and rescued the C26-induced downregulation of musclin in muscles and plasma. By contrast, musclin expression did not change in overloaded Plantaris of adult mice. Conclusions: Musclin is a myokine induced specifically by PGC1 $1 \alpha$, typically increased upon aerobic exercise and preserves muscles from wasting during C26 growth or myotubes from atrophy. Overall, musclin could be beneficial to cancer patients that cannot exercise and are at risk of developing cachexia.

\section{P. 17. The transcription factor NF-Y is required for satellite stem cell proliferation and skeletal muscle tissue repair}

Giovanna Rigillo, Valentina Basile, Silvia Belluti, Carol Imbriano

Dept. of Life Sciences, University of Modena and Reggio Emilia, Modena, Italy

Reconstruction of the skeletal muscle tissues after damage relies on the activation of Satellite Cells (SCs), a population of resident muscle stem cells. Several transcription factors are involved in SCs activation and proliferation and ensure proper temporal and spatial expression of muscle-specific genes during muscle regeneration. The transcription factor NF-Y, composed by NF-YA, NF-YB and NF-YC subunits, has an important role in the regulation of cellular proliferation and differentiation in different cell types, among which muscle cells. While NF-YA, the DNA binding subunit of NF-Y, is down-regulated in the adult muscle of WT mice, its expression is observed in the mdx mouse and correlates with euchromatic markers and expression of NF-Y target genes controlling cell growth. With the aim to investigate the role of NF-YA in the SCs proliferation and differentiation, we generated and characterized a conditional knock out mouse model in which NF-YA is deleted in Pax7+ SCs by Tamoxifen induction in adult NF-YA floxflox:Pax7 CreER mice (NF-YA cKO). Cellular and molecular analysis carried out on isolated myofibers and SCs from WT and NF-YA cKO mice highlighted that NF-Y activity is important for the maintenance of SCs homeostasis. NF-YA loss depletes Pax7+ SCs pool and impairs their proliferation. Moreover, SCs-mediated regeneration following muscle damage induced by cardiotoxin is delayed in NF-YA cKO. The effect of NF-YA abrogation was also explored in post-natal muscle growth. Immunohistological analysis showed defects in muscle morphology and a decrease in SCs number in 3 weeks aged NF-YA cKO mice, period of major increment of muscle mass by SCs-mediated myonuclear accretion. The molecular mechanism underlying the impairment of SCs activity following NF-YA loss was investigated by gene expression profiling. Overall, our results highlight a role of NF$\mathrm{Y}$ in muscle regeneration and in SCs fate, whose modulation could be useful to improve stem cell based therapies to treat muscular dystrophies.

P. 18. The Thyroid Hormone activating enzyme, Type 2 deiodinase, induces myogenic differentiation by regulating mitochondrial metabolism and reducing oxidative stress.

Serena Sagliocchi ${ }^{a}$, Annunziata Gaetana Cicatiello ${ }^{a}$, Emery Di Cicco ${ }^{a}$, Caterina Miro ${ }^{a}$, Annarita Nappia, Giuseppina Mancino ${ }^{\mathrm{a}}$, Domenico Salvatore ${ }^{\mathrm{b}}$, Monica Dentice ${ }^{\mathrm{a}}$.

a. Dept. of Clinical Medicine and Surgery - b. Dept. of Public Health, University of Naples Federico II, Naples, Italy

Thyroid hormone $(\mathrm{TH})$ is a key metabolic regulator that acts by coordinating short-term and long-term energy needs. Accordingly, significant metabolic changes are depending on thyroid status. Although it is established that hyperthyroidism augments basal energy consumption, thus resulting in enhanced metabolic state, the net effects on cellular respiration and generation of reactive oxygen species (ROS) remain unclear. To elucidate the effects of augmented TH signal in muscle cells, we generated a doxycycline-inducible cell line, in which the expression of the TH-activating enzyme, type II deiodinase (D2) is reversibly turned on by the "Tet-ON" system. Interestingly, increased intracellular TH caused a net shift from Oxidative Phosphorylation (OXPHOS) to glycolysis and a consequent increase in extracellular acidification rate. As a result, the mitochondrial ROS production, and both the basal and doxorubicin-induced production of cellular ROS were reduced. Importantly, the expression of a set of antioxidant genes was up-regulated, and, among them, the mitochondrial scavenger SOD2 was specifically induced at transcriptional level, by D2-mediated TH activation. Finally, we observed that the attenuation of the oxidative stress and increased levels of SOD2 are key elements of the differentiating cascade triggered by the TH and D2, thereby establishing that D2 is essential in coordinating metabolic reprogramming of myocytes during myogenic differentiation. In conclusion, our findings indicate that TH plays a key role in oxidative stress dynamics by regulating ROS generation. Our novel finding that $\mathrm{TH}$ and its intracellular metabolism act as mitochondrial detoxifying agents sheds new light on metabolic processes relevant to muscle physiology

P. 19. Unraveling endurance differences on dystrophy affected muscle groups. 
16th IIM Meeting (2019) - Forword \& Abstracts

Eur J Transl Myol 30 (3): 1-Yx, 2020 - doi:

Carles Sanchez-Riera, Stefano Testa, Ersilia Forneti, Cesare Gargioli, Stefano Cannata Dept. of Biology, University of Rome Tor Vergata, Italy

Muscular dystrophies are a group of heterogenous genetic neuromuscular pathologies, usually manifesting a progressive muscle degeneration accompanied by strength detriment. Despite muscular dystrophies are present in more than 40 forms, many of these affect the Dystrophin Associated Glycoprotein Complex (DAGC) being responsible for phenotype affection. Concretely, the involvement of Sarcoglycan (SG) Complex, Dystrophin, or $\alpha$-Dystroglycan, present a relative common clinical manifestation affecting proximal muscles differing on pathological severity. Nowadays, no cure is available for these disease, however different strategies are emerging against muscle degeneration likewise gene and cell therapy, or anti-inflammatory treatments. These strategies aim to reestablish the genetic disorders and muscle integrity, or at least to reduce fibrosis and stop fat replacement. Nevertheless, spontaneously several muscle groups resist degeneration and wasting process as it occurs with tongue and calf in human patients. We analyzed $\mathrm{mdx}$ and $\alpha$-sarcoglycan mouse model revealing differences on myofibers degeneration among muscle groups of the same subject, presenting common affection frequency. Our objective is to go deeper on characterization of these mice models and to look at natural strategies to overpass the genetic disease and preserve muscle tissue from degeneration and wasting process.

P. 20. Epigenetic tuning of miR in FAP-derived Extracellular Vesicles promotes regeneration and inhibits fibrosis in dystrophic muscles

Martina Sandonà ${ }^{1,2}$, Silvia Consalvi ${ }^{1,3}$, Luca Tucciarone ${ }^{1,2}$, Marco De Bardi ${ }^{1}$, Manuel Scimeca ${ }^{4,5,6}$, Daniela Angelini', Valentina Buffa ${ }^{7}$, Aadele D'Amico ${ }^{8}$, Enrico Bertini ${ }^{8}$, Sara Cazzaniga ${ }^{9}$, Paolo Bettica ${ }^{9}$, Marina Bouché $^{2}$, Antonella Bongiovanni ${ }^{7}$, Pierlorenzo. Puri ${ }^{1,3, *}$, Valentina Saccone ${ }^{1,}$

1. Istituto di Ricovero e Cura a Carattere Scientifico (IRCCS), Fondazione Santa Lucia - 2. Division DAHFMO, Unit of Histology and Medical Embryology, Sapienza University of Rome - 3. Development, Aging and Regeneration Program, Sanford Burnham Prebys Medical Discovery Institute, La Jolla - 4. Dept. of Biomedicine and Prevention, University of Rome "Tor Vergata" - 5. IRCCS San Raffaele - 6. Orchidea Lab S.r.I. - 7 Institute of Biomedicine and Molecular Immunology (IBIM), National Research Council (CNR) of Italy - 8. Unit of Neuromuscular and Neurodegenerative Disorders, Bambino Gesù Children's Hospital - 9. Clinical R\&D Italfarmaco SpA, Cinisello Balsamo.

The functional exhaustion of muscle stem cells (MuSCs) contributes to Duchenne Muscular Dystrophy (DMD) progression by compromising the compensatory regeneration of diseased muscles. MuSCs activity is influenced by functional interactions with cell types that compose their niche, including fibro-adipogenic progenitors (FAPs) that regulate the regenerative ability of skeletal muscles in physiological and pathological conditions. We have recently discovered that FAP-derived Extracellular Vesicles (EVs) support functional interactions with MuSCs, and contribute to the beneficial effect of HDAC inhibitors (HDACi) on DMD muscles. FAP-derived EVs mediate microRNA transfer to MuSCs, and that exposure of dystrophic FAPs to HDAC inhibitors (HDACi) increases the intra-EV levels of a subset of microRNAs (miRs), which cooperatively target biological processes of therapeutic interest, including regeneration, fibrosis and inflammation. In particular, increased levels of miR206 in EVs released from FAPs of muscles from DMD patients or mdx mice exposed to HDACi correlated with improvement of key histological parameters, such as compensatory regeneration and inhibition of fibrosis. We found that EVs from HDACi-treated dystrophic FAPs stimulated MuSC activation and expansion ex vivo, and promoted regeneration, while inhibiting fibrosis and inflammation of dystrophic muscles, upon intramuscular transplantation, in vivo. These data reveal a potential for pharmacological modulation of FAP-derived EV's content as novel strategy for local therapeutic interventions in muscular diseases.

\section{P. 21. Dissecting the role of Heterochromatin Conformation in Muscle Aging and Sarcopenia}

Philina Santarellia ${ }^{\mathrm{a}}$, Federica Lucinia ${ }^{\mathrm{a}}$, Jacopo Giovanni Paolo Acquasi Lozej ${ }^{\mathrm{b}}$, Gloria Pegolid, llario Tagliaferric, Sara Valsoni ${ }^{d}$, Cristiano Petrini ${ }^{\mathrm{c}}$, Endre Sebestyén ${ }^{\mathrm{c}}$, Matteo Cesarib ${ }^{\mathrm{b}}$, Luigi Solimeno ${ }^{\mathrm{b}}$, Francesco Ferraric, Chiara Lanzuoloa

a. Istituto Nazionale Genetica Molecolare Romeo ed Enrica Invernizzi, Milan - b. Fondazione IRCCS Ca' Granda, Ospedale Maggiore Policlinico, Milan - c. IFOM, the FIRC Institute of Molecular Oncology, Milan - d. Fondazione Santa Lucia IRCCS, Rome, Italy

Sarcopenia is the age-related loss of muscle mass, strength and function. Muscle mass decrease is directly responsible for functional impairment with loss of strength, increased likelihood of falls and loss of autonomy. Satellite cells are myogenic progenitors and sarcopenia is predicted to be the result of reduced satellite cell number and/or function. Despite several investigations, the precise molecular mechanisms underlying stem cell dysfunctions in the age-associated muscle decline remains unclear. Recently cutting-edge studies highlighted the role of epigenetic mechanisms in muscle senescence and sarcopenia. Among the biological mechanisms involved in the regulation of heterochromatin conformation, we recently described a functional and evolutionary conserved crosstalk between the nuclear Lamin A/C and the PcG proteins. This functional interplay is required for the maintenance of the PcG repressive functions at the onset of muscle differentiation 
16th IIM Meeting (2019) - Forword \& Abstracts

Eur J Transl Myol 30 (3): 1-Yx, 2020 - doi:

and does not work properly in the pathological Emery Dreifuss muscular dystrophy. Based on these findings we hypothesize that in aged satellite cells an altered Lamin/PcG axis could determine a pathological remodelling of the heterochromatin organization leading to the loss of muscle stem cell functions. Taking advantage of a new technique developed in our laboratory, named Sequential Analysis of MacroMolecules accessibilitY (SAMMY-seq) we were able to map lamina associated heterochromatic regions in small amount of primary cells. The protocol is based on the sequential extraction of multiple chromatin fractions, corresponding to increasingly compacted and less accessible chromatin regions, which are mapped along the genome using high-throughput sequencing. We will show results obtained in muscle tissue or stem cells derived from mice and human at different stage of muscular aging and sarcopenia with the purpose to highlight age-related chromatin structural changes, opening up the possibility to identify coding and/or noncoding genomic regions, dependent or not by Polycomb proteins, involved in premature senescence of sarcopenic muscles.

P22. Evidence supporting a morpho-functional interaction between telocytes and satellite cells in damaged skeletal muscle

Mirko Manettia, Alessia Tania, Irene Rosaa, Flaminia Chellinia, Roberta Squeccob, Eglantina Idrizaj ${ }^{b}$, Sandra Zecchi-Orlandinia , Lidia Ibba-Manneschia , Chiara Sassolia

a. Department of Experimental and Clinical Medicine, Section of Anatomy and Histology, University of Florence, Florence, Italy. b. Department of Experimental and Clinical Medicine, Section of Physiological Sciences, University of Florence, Florence, Italy.

Telocytes (TCs) represent unique stromal cells characterized by a small cell body and distinctive extremely long, thin and moniliform cytoplasmic extensions called telopodes alternating slender segments (podomers) with dilatations (podoms). TCs have been identified in the skeletal muscle interstitium with their telopodes being strategically positioned in the close vicinity of striated myofibers with regenerative features, nerve endings, small blood vessels, and often of resident muscle stem cells, namely satellite cells (SCs). A "nursing" role for TCs in SC-mediated skeletal muscle regeneration has been supposed; however, to date there is no experimental evidence demonstrating a morpho-functional interaction between these two cell types in an injured skeletal muscle. Hence, the aim of present morphological study was to explore the presence and the behavior of TCs in an ex vivo murine model of skeletal muscle (extensor digitorum longus) damage induced by forced eccentric contraction (EC) in isometric condition, focusing on their interaction with SCs. EC-damaged skeletal muscles showed evidence of structural and ultrastructural injury as judged by light microscopic and transmission electron microscopy (TEM) examination and along with significant electrophysiological changes of sarcolemnic properties. Specifically, we observed a resting membrane potential depolarization, an increase of membrane capacitance, a reduction of membrane resistance and a reduction of the outward $\mathrm{K}^{+}$current amplitude, leading to an overall alteration of myofiber excitability. TCs were identified in both control and EC-injured muscles within the interstitium, alongside the myofibers and in close vicinity of vascular structures either by CD34/CD31 double confocal immunofluorescence staining (i.e. TCs: CD34+/CD31-; endothelial cells: CD34+/CD31+) or by TEM. Of note, in EC-damaged muscles, the telopode network was more extended and arranged around activated SCs displaying nuclear positivity for Pax7, the most reliable marker of SCs, and for MyoD, the SC activation marker. TEM analysis clearly demonstrated the presence of TCs invading the SC niche passing with their telopodes a broken basal lamina to contact the underlying activated SC that exhibited a swollen appearance and an euchromatic nucleus. The interaction between TCs and SCs was confirmed by in vitro experiments performed by culturing single living endomysial sheath-covered myofibers isolated from ECdamaged muscle and the derived stromal cells/TCs and SCs. Interestingly, TCs from EC-damaged myofibers showed an increased expression of vascular endothelial growth factor (VEGF)-A, whose role in promoting myoblast proliferation and differentiation is documented. SCs isolated from the same samples exhibited an increased MyoD expression as well as a major tendency to fuse into myotubes. These findings establish for the first time a morphological interaction between TCs and SCs in a damaged muscle and suggest a juxtacrine-paracrine cell-cell interaction involving VEGF-A, which worth investigating further.

P. 23. Dissecting the possible role of $p 97$ in muscle wasting during cancer

Giulia Terribilea, Andrea David Re Cecconia, Giorgio Aquilaa, Andrea Degiorgi b, Mara Fortia, Rosanna Piccirilloa

a. Istituto di Ricerche Farmacologiche Mario Negri IRCCS, Milan, Italy - b. Università degli Studi di Parma, Italy

Half of patients with malignancies develops muscle wasting (i.e. cachexia) and aerobic exercise ameliorates their prognosis. The p97/VCP ATPase complex interacts with multiple binding proteins, estimated to be around 
16th IIM Meeting (2019) - Forword \& Abstracts

Eur J Transl Myol 30 (3): 1-Yx, 2020 - doi:

60 , and mainly extracts proteins from multimeric structures. In particular, by interacting with Ufd1 or p47, it facilitates the rapid degradation of myofibrillar proteins during muscle atrophy caused by denervation or fasting. The aim of this study was to investigate if p97 (and through which of its adaptors) plays a role also during cancer cachexia and if this is modulated by physical exercise. To induce cachexia, we injected subcutaneously one million of colon adenocarcinoma (C26) cells in BALB/c mice. This tumour causes massive muscle depletion with premature death in mice. Interestingly, by microarrays, we found that 8 out of 58 p97-binding proteins were induced in cachectic Tibialis Anterior (TA) from C26 mice, while 10 were reduced $(p<0.05)$. Further analyses of these adaptors are in progress. To understand if aerobic exercise improves cancer cachexia through p97 modulation in muscle, C26-bearing mice were run on treadmill for 5 days at $12 \mathrm{~m} / \mathrm{min}$ and $+15^{\circ}$ inclination for $45 \mathrm{~min} /$ day. By Q-PCR or Western Blotting, we measured the expression of $\mathrm{p} 97$ and its main adaptor proteins (Ufd1, Ufd2, p47) in cachectic TA muscle. In vivo, we found that the mRNA levels of p97, Ufd1, Ufd2 and p47 were induced in cachectic TA muscle from C26-carrying mice, undergoing body weight loss (i.e. cachexia). Interestingly, treadmill exercise protected C26-bearing mice from gastrocnemius muscle loss, with no effect on tumour growth, and rescued the C26-induced upregulation of p97 transcripts but not of these adaptors in muscles. Our preliminary data suggest that p97/VCP ATPase may play a role in muscle wasting also during cancer in mice. It remains to address which adaptor may be implicated and whether shRNA for $\mathrm{p} 97$ is able to recapitulate the beneficial effects of aerobic exercise in vivo.

\section{P.24 The RNA binding protein FRG1 controls transcription landscape regulating muscle maturation and metabolism.}

Antonio Vallarola ${ }^{a}$, Alberto Termanini ${ }^{a}$, Margherita Cortinia, Valeria Ghiaronia, Mattia Forcato ${ }^{a}$, Ernesto Picardib, Elena Germinario $^{c}$, Bert Blaauw ${ }^{c}$, Graziano Pesole ${ }^{b}$, Giuseppe D'Antona ${ }^{d}$, Rossella Tuplera .

a. Life Science Dept. University of Modena and Reggio Emilia - b. IBIOM-CNR, Institute of Biomembranes, Bioenergetics and Molecular Biotechnologies, National Research Council; Dept. of Biosciences, Biotechnology and Biopharmaceutics, University of Bari A. Moro - c. Biomedical Science Dept. - University of Padova - d. LUSAMMR, Laboratorio Universitario per lo Studio delle Attività Motorie nelle Malattie Rare, Sport Medicine Center - University of Pavia, Voghera, Italy

Facioscapulohumeral muscular dystrophy (FSHD), the third most common hereditary myopathy, is not due to a classical mutation within a protein-coding gene. Instead, almost all FSHD patients carry deletions of an integral number of tandem 3.3-kilobase repeat units, termed D4Z4, located on chromosome 4q35. D4Z4 deletion leads to modifications of chromatin structure and inappropriate overexpression of $4 \mathrm{q} 35$ genes. Studies have proposed several candidate genes within this genomic region and several mouse models have been generated. Among these models, mice overexpressing Facioscapulohumeral muscular dystrophy Region Gene 1 (FRG1) present a progressive myopathy that recapitulate features of human disease. FRG1 encodes for an RNA binding protein whose biological function is not well understood. To investigate the molecular mechanism triggered by FRG1 overexpression leading to overt myopathy we analyzed molecular changes occurring during disease development. At first, gene expression profiles of skeletal muscles of mice overexpressing increasing levels of FRG1 were examined at 28 days (dystrophy onset) and at 96 days (full dystrophy). We found a profound transcriptional deregulation correlating the severity of the muscle phenotype and FRG1 expression. Gene Set Enrichment Analysis and Gene Ontology revealed alterations in pathways related to muscle function, energy metabolism and inflammation. Indeed, genes associated with adult and normal myogenesis were down-regulated with a significant enrichment of genes specifically expressed during embryogenesis. By contrast, we observed the incremental activation of inflammatory pathways through time. We found that FRG1 overexpression causes the global perturbation in the mechanisms that guide postnatal muscle maturation. This process includes the anomalous expression of embryonic/neonatal proteins fundamental for muscle structures and metabolic pathways, the lack of mature proteins and the lag of muscle growth throughout postnatal life. In particular at 7 days and 14 days the expression of the embryonic isoforms of myosin remain high in FRG1 mice instead of following the physiological down-regulation occurring in WT mice, meanwhile the expression of the mature isoforms is reduced. Starting from 14 days we observed the deceleration of body weight growth curve and a reduction of muscle cross-sectional area. Moreover, FRG1 muscles displayed the significant reduction of ATP and the phosphocreatine in association with the transcriptional downregulation of Glut4, HK2 and AldoA. Our results indicate that FRG1 overexpression induce the impairment of muscle maturation and energy metabolism that precedes dystrophy. Our study opens new perspectives on the molecular mechanisms at the basis of muscular dystrophies.

P. 25. Effects of aerobic, resistance and combined exercises on 24-hr glucose variability, metabolism and muscle signalling pathways regulation in Type 1 Diabetics

Giosuè Annibalinji\#, Dean Minnock ${ }^{\text {b\# }}$, Giacomo Vallia, Serena Contarellia, Roberta Saltarellia, Carel Leroux ${ }^{c}$, Vilberto Stocchia ${ }^{\mathrm{a}}$, Elena Barbieria,d, Giuseppe De Vito ${ }^{\mathrm{b}}$; \# Equally contributing authors 
16th IIM Meeting (2019) - Forword \& Abstracts

Eur J Transl Myol 30 (3): 1-Yx, 2020 - doi:

a. Dept. of Biomolecular Sciences, University of Urbino Carlo Bo, Urbino, Italy - b. School of Public Health Physiotherapy and Sports Science, University College Dublin, Dublin 4 - c. School of Medicine, University College Dublin, Dublin 4, Ireland - d. Interuniversity Institute of Myology, Italy.

It is well known that exercise can reduce the risk of developing comorbidities and long-term complications for type 1 diabetes mellitus (T1DM) patients [1]. However, T1DM patients tend to avoid exercise to reduce the risk of hypoglycaemia [2] and losing the beneficial effects of exercise. Furthermore, T1DM patients show an impairment of the skeletal muscle growth, energy metabolism and repair mechanisms [3]. Thus, skeletal muscle has been proposed as a therapeutic target for delaying T1D complications [4] and exercise as the main tool to maintain muscle quality and function. In this study, we analysed the effect of aerobic (AER), resistance (RES) and combined (COMB) exercise in the 24-hr glucose variability and muscle signalling pathways regulation in Type 1 Diabetics. Twelve sedentary T1DM patients $(6 \mathrm{M} / 6 \mathrm{~F})$ performed on separate days and in a randomized order 40 min of high-intensity AER, RES and COMB exercise (20 min RES followed by 20 min AER). Interstitial glucose (IG) was measured with a flash glucose monitoring (FGM, Abbott FreeStyle Libre) for 24-hr pre and post exercise. The mean amplitude of glycaemic excursions (MAGE), standard deviation (SD) and coefficient variation (CV) of IG were calculated from FGM data. Muscle biopsies were collected immediately before and after each session. Myogenesis- (Myogenin, MyoD, Mrf4, Myf5), inflammation- (IL-6, TNFa, MCP1), oxidative capacity/metabolism- (PGC-1a1, PGC-1a4, GLUT-4) and muscle growth-related genes (IGF-1 isoforms) were quantified by real-time PCR. The exercise-induced skeletal muscle signalling pathways (phosphorylation of Akt, p38, ERK1/2, eEF2, AMPK) were quantified by western blot. MAGE, SD and CV decreased 24-hr post COMB. All exercise sessions reduced the CV over a 6 to12-hr period after exercise. No severe hypoglycaemic events occurred. Both RES and COMB increased the activation of the IGF-1/Akt/mTOR signalling pathway (Akt and p38MAPK phosphorylation), while eEF2 activation (dephosphorylation) and gene expression of Myogenin and MRF4 increased only after COMB and RES. Both AER and COMB promoted the splicing of IGF-1Ea variant and increased $P G C-1 \alpha$ isoforms expression. The expression of inflammation-related genes ( $T N F-\alpha, I L-6$ and $M C P-1)$ increased after all exercise sessions. Our results indicate that COMB exercise is more effective in reducing IG fluctuations compared to AER and RES alone. COMB simultaneously activates signalling related to muscular growth and oxidative capacity, which could be beneficial for short or long-term glycemic control and muscle health.

[1] Bohn B, et al, Diabetes Care. 2015 Aug;38(8):1536-43.

[2] Brazeau, AS, et al, Diabetes Care. 2008 Nov;31(11):2108-9.

[3] Coleman SK, et al, Sci Rep. 2016 Sep 1;6:32495.

[4] Forbes JM, Cooper ME, Physiol Rev. 2013 Jan;93(1):137-88.

\section{P. 26. N6-Methyladenosine as a novel pharmacological target involved in muscle regeneration \\ Costantin Heil ${ }^{a}$, Francesco Millozzi ${ }^{a}$, Luca Madaro ${ }^{b}$, Marco De Bardi ${ }^{c}$, Daniela Palacios ${ }^{a}$}

a. Laboratory of Epigenetics and Signal Transduction, IRCCS Fondazione Santa Lucia, Via del Fosso di Fiorano, 64, 00143 Rome, Italy.-

b. Epigenetics and Regenerative Medicine, IRCCS Fondazione Santa Lucia Rome, Italy - c. Neuroimmunology Unit, European Center for Brain Research (CERC), IRCCS Santa Lucia Foundation, Rome, Italy.

Duchenne muscular dystrophy (DMD) is a neuromuscular disease caused by a deficiency of the dystrophin protein. Currently no cure is available for the disease and numerous pharmacological approaches are being studied to extend the regenerative phase of dystrophic muscles. In this project we aim to investigate if the modulation of the epi-transcriptome could represent a new pharmacological tool for DMD. In recent years, genetic and biochemical studies have revealed how post-transcriptional modifications of RNA influence various cellular mechanisms, in particular splicing, miRNA processing and transcripts stability. One of the most important changes in RNA is N6-methyladenosine (m6A). This modification is added from a complex containing the METTL3 / METTL14 heterodimer and removed from RNA demethylases such as FTO and ALKBH5. We have recently observed an increase in m6A levels in skeletal muscles derived from dystrophic mice compared to wilde type animals, suggesting that under pathological conditions the N6-Methyladenosine modification could influence muscle regeneration through the modulation of various cellular pathways. To validate this hypothesis, we conducted several experiments both in vivo and ex vivo using pharmacological and genetic approaches, aimed at modulating the expression and activity of components of the methylation machinery. In the pharmacological approach we have treated dystrophic mice with a drug approved by the FDA that has recently shown to increase RNA methylation through the inhibition of FTO demethylase; the genetic approach instead involves the use of siRNA against Metll3 in primary myoblasts. Our preliminary results showed that the RNA methylome $(\mathrm{m} 6 \mathrm{~A})$ is dynamically modulated during skeletal muscle differentiation and regeneration, pointing at the epi-transcriptome as a new potential target for therapeutic interventions. In addition, we identified new METTL3 direct targets in muscle cells through RNA-seq experiments. Finally, increasing RNA methylation by FTO inhibition modulates the regenerative response in dystrophic mice. Altogether, the results derived from these studies will set up the basis for a new therapy based on the modulation of the epi-transcriptome for DMD. 
16th IIM Meeting (2019) - Forword \& Abstracts

Eur J Transl Myol 30 (3): 1-Yx, 2020 - doi:

P. 27. Identification of a phytotherapy formulation to counteract muscle atrophy

Laura Salvadoria,d, Manuela Mandrone ${ }^{\mathrm{b}}$, Tommaso Manentic ${ }^{\mathrm{c}}$, Catia Ercolanic, Luca Corniolic, Maria Caterina Lianza $^{b}$, Paola Tomasi ${ }^{b}$, Ferruccio Polib, Guglielmo Sorcia,d and Francesca Riuzzia,d

a. Dept. Experimental Medicine, University of Perugia, Italy - b. Dept. Pharmacy and Biotechnology, University of Bologna, Italy -

c. Biokyma Laboratory srl, Anghiari, Italy - d. Interuniversity Institute of Myology (IIM), Perugia, Italy

Skeletal muscle atrophy is a loss of muscle mass and strength associated with several pathologies including diseases characterized by systemic chronic inflammation and prolonged treatment with glucocorticoids (GCs) $[1,2]$. Despite it represents an enormous medical problem that accelerates the disease progression, increases hospitalization, and worsens quality of patients' life, an effective therapy to muscle atrophy is still lacking. The increase of a catabolic state resulting in the breakdown of myofibrillary proteins, especially myosin heavy chain $(\mathrm{MyHC})$, is recognized as the main process promoting muscle atrophy [3]. Recently, the interest in plant extracts/metabolites active in the pathophysiology of muscle is obtaining growing attention [4]. Starting from one hundred hydroalcoholic extracts from medical plants, our work was aimed to individuate a herbal formulation efficient in counteracting muscle atrophy. By using well-characterized in vitro experimental models mimicking muscle atrophy, i.e., treatment of myotubes from $\mathrm{C} 2 \mathrm{C} 12$ myoblasts with proinflammatory cytokines (TNFa/IFNy) or excess GCs (dexamethasone) [3], we firstly identified fifteen extracts able to rescue myotube atrophy, by morphological analysis after May-Grunwald/Giemsa staining. Ten extracts displayed an extremely potent protective effect against myotube atrophy and $\mathrm{MyHC}$ degradation, as indicated by measurement of myotube diameters after immunofluorescence staining for MyHC-lla, even if at different extent in the presence of cytokines or dexamethasone. Based on their anti-atrophic potential and NMR-based metabolic profiles, we selected six extracts (Withania somnifera Dunal, Panax ginseng Meyer, Silybum marianum Gaertner, Peumus boldus Molina, Trigonella foenum-graecum $L$. and Urtica dioica $L$.) to obtain twenty combinations of three extracts to test on myotube cultures. Three herbal formulations showed a surprising ability to counter the reduction of myotube diameters and degradation of $\mathrm{MyHC}$ in the presence of different atrophic stimuli. Our results might lead to the development of a low-cost, non-toxic phytotherapy product able to prevent/counteract muscle atrophy associated with different pathological conditions.

1) Dutt $V$ et al., Pharmacol Res. 2015, 46:86-100

2) Schakman O et al., Int J Biochem Cell Biol. 2013, 45:2163-72

3) Bonaldo P, Sandri M. Dis Model Mech. 2013, 6:25-39

4) Jeong SM et al., Evid-Based Complement Alternat Med. 2018, ID:8654719 